\title{
Elastic moduli tensors, ideal strength, and morphology of stanene based on an enhanced continuum model and first principles
}

\author{
Hossein M. Shodja ${ }^{\mathrm{a}, \mathrm{b}, *}$, Farzaneh Ojaghnezhad ${ }^{\mathrm{c}}$, Azadeh Etehadieh $^{\mathrm{a}}$, \\ Maryam Tabatabaei ${ }^{a}$ \\ ${ }^{a}$ Department of Civil Engineering, Sharif University of Technology, P.O. Box \\ 11155-4313, Tehran, Iran \\ ${ }^{b}$ Institute for Nanoscience and Nanotechnology, Sharif University of Technology, P.O. \\ Box 11155-9161, Tehran, Iran \\ ${ }^{c}$ School of Engineering, Alzahra University, Vanak St., 1993891176, Tehran, Iran
}

\begin{abstract}
The present work aims to provide an accurate description of the tensile behavior of the planar as well as low-buckled stanene and to capture their ideal strength in armchair (AC)- and zigzag (ZZ)-directions. For an accurate description of anisotropic response of such hyperelastic materials as stanene, consideration of a highly nonlinear constitutive model in which up to the fourth power of strains is incorporated is inevitable. By utilizing first principles calculations based on density functional theory (DFT), the second, third, fourth, and fifth order elastic moduli tensors corresponding to both planar and low-buckled states are obtained. Moreover, the morphology of the freestanding stanene such as bond length and lattice parameter is determined; for low-buckled stanene two additional parameters, namely, buckling height and dihedral angle are computed. The effects of uniaxial and biaxial loadings
\end{abstract}

\footnotetext{
${ }^{*}$ Corresponding author

Email address: shodja@sharif.edu (Hossein M. Shodja)
} 
germane to AC- and ZZ-directions on the buckling height and dihedral angle are also studied. Scrutinization of the electronic charge distribution reveals the phenomenon of the formation of necking in the Sn-Sn bonds under large uniaxial extension along the AC-direction. Moreover, the transformation of $s p^{3}$ hybridized orbitals to $s p^{2}$ hybridized orbitals is examined and confirmed through consideration of the structural geometries of the atomic bond angles. Keywords: stanene, nonlinear continuum, morphology, elastic moduli tensors, ideal strength, ab-initio

\section{Introduction}

Ever since graphene has been introduced to the scientific world, it has attracted the attention of many researchers with diverse backgrounds. For the sake of achievable technological applications, abundant attention has been absorbed in graphene due to its unusual electronic properties arising from the $s p^{2}$ hybridized planar structure (Manjanath et al., 2014). Motivated by the intriguing electronic and optoelectronic properties of free-standing graphene, many researches have been made for probing alternative two-dimensional (2D) materials. Theorists predict that new monolayer materials with more better electronic properties than those of graphene can be fabricated being capable to be easily integrated with current generation of electronic technologies (Balendhran et al., 2015). Moreover, it is expected that advanced opportunities in nanoelectronic devices and energy technologies are triggered by these monolayer materials (Zhuang and Hennig, 2014). Recent studies show that more interesting phenomena are observed by proceeding down in group IV of the Periodic Table (Gross, 2014). In the hypothetical field of 
room-temperature superconductors, for example, monolayer stanene may act as zero-heat-loss interconnects in processor chips and hence may put an end to the one-century tantalization of engineers by physicists for superconductors (Webb, 2014).

In contrast to graphene which is stable in planar configuration, stanene is stable in low-buckled configuration. This is associated to the fairly weak strength of $\pi-\pi$ bonding between tin atoms. Buckling increases the overlap between $\pi$ and $\sigma$ bondings resulting in stability of low-buckled stanene (Xu et al., 2013). Similar to its 2D group-IV counterpart, silicene, the out-ofplane buckling of stanene makes it possible to be functionalized by an outof-plane electric field. Since the buckling of stanene is larger than that of silicene, it is expected that a larger band gap is induced. (Drummond et al., 2012; Van den Broek et al., 2014). Topological insulators (TIs) which behave as an ordinary insulator in three-dimensional (3D) configuration and have gapless surface (or edge) states in two-dimensional (2D) configuration have attracted great attention in condensed matter physics and materials science (Hasan and Kane, 2010; Xu et al., 2013). In special elements for which large spin-orbit (SO) coupling exists, the usual band ordering is inverted and a topologically trivial insulator can be converted into a quantum spin hall (QSH) insulator. Xu et al. (2013) and Liu et al. (2011) have observed that the presence of SO interaction results in QSH state in stanene. Recently, we have also obtained the phenomenon of band inversion in both strained and unstrained states of stanene due to SO coupling. Moreover, we found that lattice distortion at special strain level of 0.4 along armchair direction can play the key role in the transformation of a $2 \mathrm{D}$ semi-metallic state to 
nontrivial QSH insulator for stanene (Tabatabaei et al., 2016). In comparison to graphene with extremely small opened gap (Min et al., 2006; Yao et al., 2007), the unstrained stanene has a band gap of about $0.115 \mathrm{eV}$ which is tunable using strain loading (Tabatabaei et al., 2016). In addition to the application of strain loading to modulate the band gap of stanene, Zhang et al. $(2015,2016)$ showed that it can also be tuned by functionalization. Stanene as a large-gap quantum spin Hall insulator is expected to find a wide range of applications in development of room-temperature dissipationless conduction, spintronic devices, and highly miniaturized electronics $(\mathrm{Xu}$ et al., 2013; Zhu et al., 2015; Song et al., 2014). It is notable to mention that incorporation of the room-temperature dissipationless conductors in appropriate sections of integrated circuits and microprocessors would significantly cut down on the power consumption and heat production. The hope for practicability of these intriguing applications has enormously increased after the successful fabrication of stanene on $\mathrm{Bi}_{2} \mathrm{Te}_{3}(111)$ substrates by molecular beam epitaxy as recently reported by Zhu et al. (2015). They have determined the atomic and electronic structures of epitaxial stanene by scanning tunneling microscopy and angle-resolved photoemission spectroscopy in combination with first principles calculations. Their experimental observations are in a reasonable agreement with the previous theoretical predictions. More interestingly, Saxena et al. (2016) have recently synthesized one-atom thick free-standing layer of stanene.

Determination of the mechanical properties of novel materials, due to its fundamental importance, has been the subject of numerous works in the literature. Regarding the possible vast applications, the mechanical properties 
of such a novel material as stanene are critical in designing the associated structures. Surprisingly, a brief surveying the publications on stanene shows that neither the nonlinear elastic behavior of stanene nor its intrinsic stress is addressed in the literature. Owing to the computationally power-intensive feature of simulation methods as well as the reasonable mechanical predictions of the continuum approaches in nanoscale problems (Delfani et al., 2013), the development of the continuum framework for stanene would be of great benefit to predict its in-plane elastic response in nano-devices. The nonlinear elastic behavior of some monolayer materials up to the ultimate stress followed by a strain softening until fracture are previously studied by some researchers (Topsakal et al., 2010; Peng et al., 2012, 2013; Wei et al., 2009; Delfani et al., 2013). To develop the continuum framework, the higher order series expansion of the strain energy density function of $2 \mathrm{D}$ stanene with respect to the Lagrangian strain is considered. Subsequently, the enhanced continuum constitutive relations are written in terms of the higher order elastic moduli tensors. The higher order elastic constants are evaluated by fitting the nonlinear stress-strain analytical expressions to the corresponding ab-initio data for appropriate number of loading modes. Ab-initio simulations are carried out employing Vienna Ab-initio Simulation Package (VASP) based on pseudopotential density functional theory (DFT). The most mentionable advantages of the first principles calculations are their high transferability and quantitative reliability. The total energies of the system, forces on each atom, and stresses on the simulation box can be directly obtained from DFT calculations. By numerical values of the higher order elastic constants on hand, one can study the nonlinear elastic response of the ma- 
terial in uniaxial tensile stress up to some point beyond its intrinsic stress. In this way, the ideal strength of stanene can be determined in zigzag (ZZ)and armchair (AC)-directions. DFT calculations can be employed to assess the ideal strengths for validation of the continuum model, as well. Moreover, the in-plane Young's modulus and Poisson's ratio can be calculated via the second order elastic constants of the material. In this work, stanene is considered as a continuous lamina with non-zero thickness. We assume that the deformed state of stanene is such that the contribution of bending to the strain energy density is negligible as compared to the in-plane strain contribution. This requires that the radius of curvature of any out-of-plane deformation be significantly larger than the in-plane interatomic distance. The stress state of stanene under these assumptions can be assumed to be in-plane (Fumi, 1952).

The paper is organized as follows. Details of the atomic structure of stanene is presented in Section 2. Section 3 is devoted to illustrate the nonlinear elastic continuum description and the fifth order series expansion of the strain energy density function. In Section 4, the details of employed methodologies for determination of the higher order elastic constants are presented. The detailed explanations are given for both analytical exploited approaches such as stress-strain and energy-strain methods and the ab initio computational simulations. In Section 5, the methods for calculation of ideal tensile strength are described in detail. The first method is based on an analytical model in the nonlinear continuum framework (Section 5.1) and the next one is a computational approach including uniaxial tension simulation in VASP (Section 5.2). The numerical results and pertinent discussions 
are reported in Section 6. In Section 6.1, the morphological properties are given and compared with the existing data in the literature. The values of the higher order elastic constants for planar stanene, buckled stanene, and graphene are tabulated in Section 6.2. Section 6.3 represents the numerical values for ideal strength of the considered materials, and in Section 6.4 the values for Young's modulus and Poisson's ratio are given.

\section{Atomic structure of stanene}

The geometry of the monolayer tin film called stanene is depicted in Fig. 1. It is well known that stanene is a 2D hexagonal lattice of the crystal class, $D_{6 h}$ possessing a six-fold rotation axis and six mirror planes perpendicular to its plane. Assume that the Cartesian axes $x_{1}$ and $x_{2}$ coincide with the AC and ZZ directions of stanene, respectively, as shown in Fig. 1. The rhomboid two-atomic unit cell of stanene is constructed via the lattice vectors, $\boldsymbol{v}^{(1)}=$ $\frac{\sqrt{3}}{2} a_{0} \boldsymbol{i}+\frac{1}{2} a_{0} \boldsymbol{j}$ and $\boldsymbol{v}^{(2)}=a_{0} \boldsymbol{j}$ where $a_{0}$ is the equilibrium lattice parameter of stanene. The third lattice vector of the unit cell, $\boldsymbol{v}^{(3)}$ is assumed to be perpendicular to the plane of the monolayer.

Recent studies have shown that some novel monolayers such as silicene (Roman and Cranford, 2014) and stanene (Xu et al., 2013) are more stable in their buckled configuration than their planar one. Hence, both buckled and planar configurations are essential to be perused. Stanene is a one-atomthick layer and can be subjected to large elastic deformations. Thus, for an accurate description of the mechanical properties of both its aforementioned states, an enhanced constitutive relation in which fourth through tenth order elastic moduli tensors (second through fifth order in Voigt notation) are 


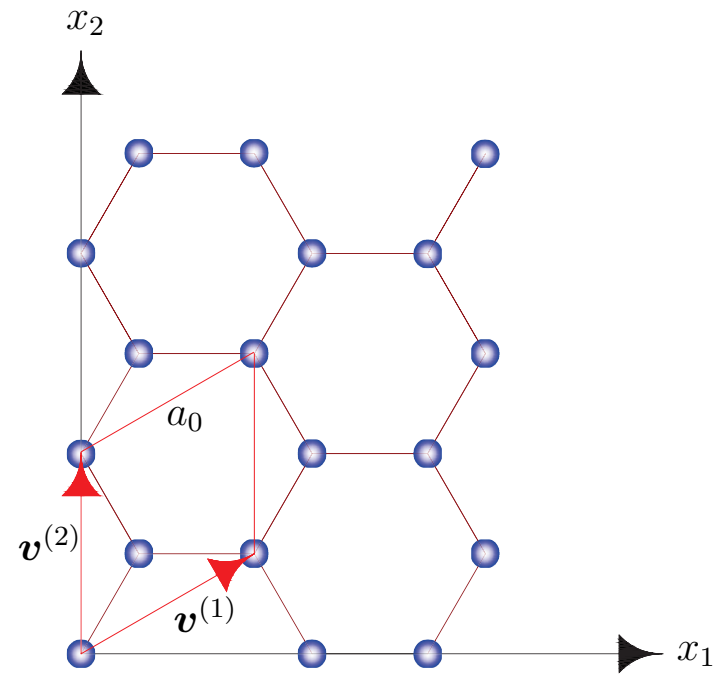

Figure 1: The atomic structure of stanene and the corresponding in-plane lattice vectors, $\boldsymbol{v}_{1}$ and $\boldsymbol{v}_{2}$ and lattice parameter, $a_{0}$. AC- and ZZ-directions of the monolayer coincide with $x_{1}$ and $x_{2}$ axes, respectively. The two-atomic rhomboid unit cell formed by the lattice vectors $\boldsymbol{v}_{1}$ and $\boldsymbol{v}_{2}$ is shown.

accounted for is considered. Subsequently, the corresponding elastic moduli tensors as well as the ideal strength is calculated with the aid of ab-initio DFT. In this work, this is achieved using two different approaches of stressstrain relation and energetic considerations. Recently, Wei et al. (2009) obtained the elastic moduli tensors of graphene using stress-strain relation. For the sake of comparison, in addition to the stress-strain relation, we employ energy approach for the calculation of the elastic moduli tensors and the ideal strength of graphene. As it will be seen the energy approach yields values closer to the available experimental results. As expected, the energetic consideration is more accurate; the stress-strain approach, requires the additional processes of differentiation of the energy which can accompany some inaccuracies. Employing the first principles modeling, such geometrical parameters as the lattice parameter, buckling height, bond length, and dihedral angle are also calculated. 


\section{Constitutive relation}

For an accurate prediction of the mechanical behavior of $2 \mathrm{D}$ materials subjected to finite deformation, knowledge of an appropriate constitutive equation that describes the stress-strain relationship precisely is inevitable. For example, in order to capture the ideal strength of single-walled carbon nanotubes, Delfani and Shodja (2013) utilized a highly nonlinear constitutive relation associated with graphene. This relation is derived from a strain energy function in which the terms consisting the second through fifth powers of the strain field have been included. In Lagrangian description, the energy density function, $W(\boldsymbol{\eta})$ for such hyperelastic materials as stanene in terms of the Lagrangian strain tensor, $\boldsymbol{\eta}$ may be expressed as

$$
\begin{aligned}
W & =\frac{1}{2 !} C_{I J} \eta_{I} \eta_{J}+\frac{1}{3 !} C_{I J K} \eta_{I} \eta_{J} \eta_{K}+\frac{1}{4 !} C_{I J K L} \eta_{I} \eta_{J} \eta_{K} \eta_{L} \\
& +\frac{1}{5 !} C_{I J K L M} \eta_{I} \eta_{J} \eta_{K} \eta_{L} \eta_{M},
\end{aligned}
$$

where Voigt notation (Nye, 1985) has been employed. In the above relation, the summation convention is adopted for the repeating uppercase Voigt indices. Voigt indices run from 1 to 6 and correspond to 11, 22, 33, 23, 13 , and 12 , respectively. The constants $C_{I J}, C_{I J K}, C_{I J K L}$, and $C_{I J K L M}$ are, respectively, the components of the second, third, fourth, and fifth order elastic moduli tensors in the context of Voigt notation. In the usual Cartesian notation, these are the fourth, sixth, eighth, and tenth order elastic moduli tensors, respectively. For the description of the mechanical behavior of stanene in the linear range where the strains are quite small, the first term in Eq. (1) suffices; however, to describe its nonlinear behavior and obtain its ideal strength, one must maintain all the terms in Eq. (1). Due to the 
fact that stanene is a $2 \mathrm{D}$ hexagonal lattice of the crystal class, $D_{6 h}$ having a six-fold symmetry, the only non-zero components (indices) of the aforementioned elastic moduli tensors span over 1, 2, and 6. Under these conditions, the second through fifth order elastic moduli tensors consist of fourteen independent constants. A brief discussion on this issue is provided in Appendix A. The method of calculation of these constants using stress-strain relation combined with ab initio DFT is given in section 4.1. The outline of the approach involving energy consideration is provided in section 4.2.

\section{Methodologies of the evaluation of the elastic constants using ab-initio simulation}

An accurate description of the elastic constants of buckled stanene using first principles calculations is of particular interest. For the sake of comparison, the constants pertinent to the planar stanene are also calculated. Such 2D materials as stanene and graphene can undergo large elastic deformations. In order to capture the ideal tensile strength of stanene analytically with reasonable accuracy, the knowledge of the numerical values of the components of the second through fifth order tensors (in Voigt notation) are required. Delfani and Shodja (2013) have noted that for providing an enhanced continuum modeling of the ideal strength of single-walled carbon nanotubes, the knowledge of the second through fifth order tensors for the corresponding graphene is necessary. The continuum mechanics approach as well as first principles calculations approach to the determination of the ideal strength of stanene will be given in the next section.

By consideration of different modes of strain loading and with the aids of 
the first principles calculations, one can evaluate the components of the elastic moduli tensors. The theoretical approach to the evaluation of the elastic properties of materials is fairly routine and well-understood. Nonetheless, the existence of such data in the literature for various substances is quite useful for further studies of their mechanical responses due to different modes of deformations and loadings. Several recent theoretical contributions on the estimation of the elastic properties of different types of materials include the works of Wei et al. (2009); Ojaghnezhad and Shodja (2012); Delfani et al. (2013); Peng et al. (2012, 2013); Shodja et al. (2013, 2014), among others. To extract the elastic constants of stanene, several special deformation states are considered. For self-containment, a brief description for obtaining these constants is given herein.

\subsection{Approach based on stress-strain relation}

The second Piola-Kirchhoff $(\mathrm{P}-\mathrm{K})$ stress tensor, $\Sigma_{I}$ in terms of its work conjugate Lagrangian strain, $\eta_{I}$ is readily obtained via $\Sigma_{I}=\partial W / \partial \eta_{I}$ as

$$
\Sigma_{I}=C_{I J} \eta_{J}+\frac{1}{2 !} C_{I J K} \eta_{J} \eta_{K}+\frac{1}{3 !} C_{I J K L} \eta_{J} \eta_{K} \eta_{L}+\frac{1}{4 !} C_{I J K L M} \eta_{J} \eta_{K} \eta_{L} \eta_{M} .
$$

With reference to Fig. 1, a state of uniaxial strain in the $x_{1}$-direction is characterized by $\eta_{1} \neq 0$ and $\eta_{2}=\eta_{6}=0$. The corresponding elastic response gives $\Sigma_{1} \geq 0, \Sigma_{2} \geq 0$, and due to symmetry, $\Sigma_{6}=0$; note that for this configuration, the value of the lateral stress is adjusted in such a way that the lateral strain is kept identically equal to zero. Next, a state of uniaxial strain in the $x_{2}$-direction is employed in a similar manner. Finally, a state of biaxial strain for which $\eta_{1}=\eta_{2}=\eta \neq 0$ and $\eta_{6}=0$ is applied, which results in $\Sigma_{1}=\Sigma_{2} \geq 0$ and $\Sigma_{6}=0$. From Eq. (2), the components of the second 
Table 1: The relations for non-zero components of the second P-K stress tensor in different modes of loadings.

\begin{tabular}{ll}
\hline Modes of loadings & $\Sigma_{1}(\eta)$ and $\Sigma_{2}(\eta)$ \\
\hline$\eta_{1}=\eta, \eta_{2}=\eta_{6}=0$ & $\Sigma_{1}=C_{11} \eta_{1}+\frac{1}{2} C_{111} \eta_{1}^{2}+\frac{1}{6} C_{1111} \eta_{1}^{3}+\frac{1}{24} C_{11111} \eta_{1}^{4}$ \\
$\Sigma_{2}=C_{12} \eta_{1}+\frac{1}{2} C_{112} \eta_{1}^{2}+\frac{1}{6} C_{1112} \eta_{1}^{3}+\frac{1}{24} C_{11112} \eta_{1}^{4}$ \\
$\Sigma_{1}=C_{12} \eta_{2}+\frac{1}{2}\left(C_{111}-C_{222}+C_{112}\right) \eta_{2}^{2}+\frac{1}{12}\left(C_{1111}+2 C_{1112}-C_{2222}\right) \eta_{1}^{3}$ \\
$\Sigma_{2}=C_{11} \eta_{2}+\frac{1}{2} C_{222} \eta_{2}^{2}+\frac{1}{6} C_{2222} \eta_{2}^{3}+\frac{1}{24} C_{22222} \eta_{2}^{4}$ \\
$\eta_{1}=\eta_{1}=0 \quad \Sigma_{2}=\left(C_{12}+C_{11}\right) \eta+\frac{1}{2}\left(2 C_{111}-C_{222}+3 C_{112}\right) \eta^{2}$ \\
$+\frac{1}{6}\left(3 / 2 C_{1111}+4 C_{1112}-1 / 2 C_{2222}+3 C_{1122}\right) \eta^{3}$ \\
$+\frac{1}{24}\left(3 C_{11111}+10 C_{11112}-5 C_{12222}+10 C_{11122}-2 C_{22222}\right) \eta^{4}$
\end{tabular}

P-K stress tensor for the three above-mentioned modes of strain loadings are readily derived as displayed in Table 1 .

As it was alluded to, the constitutive relation $W(\boldsymbol{\eta})$ given by Eq. (1) involves fourteen independent constants all of which have appeared in the stress-strain relations given in Table 1. It is observed that all of the fourteen constants can be evaluated by the consideration of three modes of loading.

\subsection{Approach based on energy consideration}

For the calculation of the constants via energetic consideration, by employing the modes of loadings mentioned in the previous subsection, we can only compute the non-zero elements of the second through third order elastic moduli tensors. In order to compute the remaining constants, consideration of two additional modes of loadings are required. These two additional modes of strain loadings as well as the previous three modes and pertinent expressions for the strain energy density are included in Table 2. Since the stresses are obtained through differentiation of $W(\boldsymbol{\eta})$ with respect to $\boldsymbol{\eta}$, the energy approach undertaken in the present work is expected to lead to a more ac- 
Table 2: The relations for the strain energy density of 2D material in five different modes of loadings.

\begin{tabular}{ll}
\hline Modes of loadings & $\mathrm{W}(\eta)$ \\
\hline$\eta_{1}=\eta, \eta_{2}=\eta_{6}=0$ & $\frac{1}{2} C_{11} \eta^{2}+\frac{1}{6} C_{111} \eta^{3}+\frac{1}{24} C_{1111} \eta^{4}+\frac{1}{120} C_{11111} \eta^{5}$ \\
$\eta_{2}=\eta, \eta_{1}=\eta_{6}=0$ & $\frac{1}{2} C_{11} \eta^{2}+\frac{1}{6} C_{222} \eta^{3}+\frac{1}{24} C_{2222} \eta^{4}+\frac{1}{120} C_{22222} \eta^{5}$ \\
$\eta_{1}=\eta_{2}=\eta, \eta_{6}=0$ & $\left(C_{11}+C_{12}\right) \eta^{2}+\frac{1}{3}\left(2 C_{111}+3 C_{112}-C_{222}\right) \eta^{3}$ \\
& $+\frac{1}{24}\left(3 C_{1111}+8 C_{1112}+6 C_{1122}-C_{2222}\right) \eta^{4}$ \\
& $+\frac{1}{60}\left(3 C_{11111}+10 C_{11112}+10 C_{11122}-5 C_{12222}-2 C_{22222}\right) \eta^{5}$ \\
& $\left(C_{11}-C_{12}\right) \eta^{2}+\frac{2}{3}\left(C_{111}-C_{222}\right) \eta^{3}$ \\
& $-\frac{1}{24}\left(C_{1111}+8 C_{1112}-6 C_{1122}-3 C_{2222}\right) \eta^{4}$ \\
& $-\frac{1}{30}\left(C_{11111}+5 C_{11112}-5 C_{12222}-C_{22222}\right) \eta^{5}$ \\
& $\frac{1}{4}\left(5 C_{11}+3 C_{12}\right) \eta^{2}+\frac{1}{12}\left(8 C_{111}+9 C_{112}-C_{222}\right) \eta^{3}$ \\
& $+\frac{1}{384}\left(71 C_{1111}+120 C_{1112}+54 C_{1122}+11 C_{2222}\right) \eta^{4}$ \\
& $+\frac{1}{960}\left(53 C_{11111}+120 C_{11112}+90 C_{11122}-15 C_{12222}+8 C_{22222}\right) \eta^{5}$ \\
\hline$\eta_{1}=\eta_{2}=\eta_{6}=\eta$ &
\end{tabular}

curate result than that obtained from the stress-strain relation.

\subsection{Ab initio simulation}

By utilizing DFT calculations, the elastic response of stanene for different modes of loadings noted in Tables 1 and 2 is obtained. DFT calculations are carried out via VASP (Kresse and Hafner, 1994, 1993; Kresse and Furthmuller, 1996a,b) employing the projector augmented wave method (Blochl, 1994) and the generalized gradient approximation (GGA) (Perdew et al., 1996, 2008). The calculations are performed at absolute zero temperature. Note that the employed ab-initio simulation package imposes periodic boundary conditions to the unit cell along the direction of the lattice vectors. Hence, to simulate the two-atom unit cell of stanene (Fig. 1), it is necessary to add a sufficient number of additional layers of vacuum over the two 
faces of stanene sheet so that the periodically distributed stanene sheets are not interacting. In this work, the distance between the two adjacent layers is taken to be about $\left|\boldsymbol{v}^{(3)}\right|=3.5 a_{0}$ in which $a_{0}$ is approximately $4.8 \AA$ according to Manjanath et al. (2014). The irreducible Brillouin Zone is sampled with a Gamma-centered $11 \times 11 \times 1$ grid of k-points and, moreover, a plane-wave cutoff of $500 \mathrm{eV}$ is used in all calculations. The criterion to stop the relaxation of the electronic degrees of freedom is satisfied when the total energy change between two consecutive electronic steps is smaller than $10^{-6} \mathrm{eV}$. The optimized ionic geometry is achieved when the change in the total free energy between two consecutive ionic steps is smaller than $0.001 \mathrm{eV}$. To obtain the equilibrium lattice parameter of stanene, $a_{0}$, the total energy of the unit cell for various values of $a_{0}$ is computed through relaxation of the Sn atoms into their instantaneous ground state using conjugate gradient algorithm. By plotting the total energy versus the lattice parameter, the equilibrium lattice parameter corresponding to the minimum total energy is obtained.

The stresses computed by VASP are the components of true or Cauchy stress tensor in units of force per current area associated with the deformed unit cell. For a 2D material, however, it is necessary to express the stress in terms of force per unit length. Thus, the stress components are obtained through multiplying the stresses calculated by VASP by the interlayer spacing of $16.8 \AA$. The relation between the true stress, $\boldsymbol{\tau}$ and the second P-K stress, $\boldsymbol{\Sigma}$ is given as (Malvern, 1969)

$$
\boldsymbol{\Sigma}=J \boldsymbol{F}^{-1} \cdot \boldsymbol{\tau} \cdot\left(\boldsymbol{F}^{-1}\right)^{T}
$$

where $J$ is the determinant of the deformation gradient tensor, $\boldsymbol{F}$. Recall that the deformation tensor, $\boldsymbol{D}$ and the deformation gradient, $\boldsymbol{F}$ are related 
by

$$
\boldsymbol{D}=\boldsymbol{F}-\boldsymbol{I}
$$

where $\boldsymbol{I}$ is the identity tensor. The Lagrangian strain tensor, $\boldsymbol{\eta}$ can then be expressed in terms of $\boldsymbol{D}$ as

$$
\boldsymbol{\eta}=\frac{1}{2}\left(\boldsymbol{D}+\boldsymbol{D}^{T}+\boldsymbol{D}^{T} \cdot \boldsymbol{D}\right)
$$

The primitive lattice vectors of the deformed configuration, $\tilde{\boldsymbol{v}}^{(i)}, i=1,2$ are related to those of the reference (undeformed) configuration, $\boldsymbol{v}^{(i)}$ via

$$
\tilde{\boldsymbol{v}}^{(i)}=\boldsymbol{v}^{(i)}+\boldsymbol{D} \cdot \boldsymbol{v}^{(i)}, \quad i=1,2 .
$$

To calculate the elastic response of stanene in each deformation state, the strains are imposed to the unit cell by changing the lattice vectors, $\boldsymbol{v}^{(i)}$ to the deformed lattice vectors, $\tilde{\boldsymbol{v}}^{(i)}$ according to Eq. (6). The tin atoms are then relaxed in the strained unit cell; so that the total energy of the system is a minimum. The strain is employed in small increments beginning with the initial unloaded unit cell through the final stage of the loading prior to the occurrence of elastic instability in the system.

By exploiting the first principles molecular dynamics simulation, Van den Broek et al. (2014) have shown that stanene has a phonon dispersion without imaginary frequencies, and hence, phonon instability would not occur for this material. Nevertheless, for the evaluation of the elastic constants in the framework of continuum mechanics, it is required that the material remains elastic during the strain loadings. For large strains, to make sure that the response is elastic, the unit cell is unloaded to verify that the unloading path coincides with that of the loading. For example, Shodja et al. (2014) have 
employed DFT calculations by VASP to determine the threshold point of inelastic behavior for amorphous silicon.

By evaluating the elastic response of stanene in each mode of strain loading, the components of the second through fifth order elastic moduli tensors can be obtained by either energetics or stress-strain relations. In the energy approach, the expressions for the strain energy density based on continuum theory given in Table 2 are fitted to the pertinent data obtained from VASP. In the approach based on the stress-strain relation, the stress-strain relations given in Table 1 for different modes of loadings are fitted to the second P-K stress-strain data obtained from VASP.

\section{Methodologies of the calculation of the ideal strength in the AC- and ZZ-directions}

The ideal tensile strength is an inherent material property which is strongly influenced by the atomic structure and bond quality, and thus, its prediction a priori is a complicated matter. The theoretical or ideal tensile strength is the maximum stress that a solid can endure just before exhibiting its first internal instability and entering the phase of plastic deformation (Shodja et al., 2014). Morris and Krenn (2000) and Roundy et al. (2001) refer to the ideal strength as the stress at elastic instability. At strains larger than that corresponding to the ideal strength, the monolayer lattice becomes unstable and ruptures spontaneously. Calculation of the ideal tensile strength of stanene in its natural buckled configuration is of particular interest. This will be done by two different methods. The first approach is based on continuum theory which utilizes the enhanced constitutive relation (1). In the 
second approach ab initio DFT is employed, and subsequently, the results are compared.

\subsection{Approach based on the proposed enhanced constitutive relation}

In order to capture the stress-strain behavior of stanene and subsequently its ideal strength with high accuracy in the framework of continuum mechanics, utilization of the fourth, sixth, eighth, and tenth order elastic moduli tensors in the corresponding constitutive relation is inevitable. We are in particular interested in calculating the ideal strength of stanene for two cases of uniaxial tension in the AC- and ZZ-directions. Thus, in both cases the strain components $\eta_{1}$ and $\eta_{2}$ are non-zero and, moreover, due to six-fold symmetry of stanene no shearing strain is induced (i.e., $\eta_{6}=0$ ). By using Eq. (2), the components of the second $\mathrm{P}-\mathrm{K}$ stress tensor, $\Sigma_{I}, I=1$, 2 , respectively, along AC- and ZZ-directions in terms of $\eta_{1}$ and $\eta_{2}$ are given as

$$
\begin{aligned}
\Sigma_{1} & =C_{11} \eta_{1}+C_{12} \eta_{2}+\frac{1}{6} C_{1111} \eta_{1}^{3}+\frac{1}{2} C_{111} \eta_{1}^{2}+\frac{1}{2}\left(C_{111}+C_{112}-C_{222}\right) \eta_{2}^{2} \\
& +C_{112} \eta_{1} \eta_{2}+\frac{1}{12}\left(C_{1111}+2 C_{1112}-C_{2222}\right) \eta_{2}^{3}+\frac{1}{2} C_{1112} \eta_{1}^{2} \eta_{2} \\
& +\frac{1}{24} C_{11111} \eta_{1}^{4}+\frac{1}{24} C_{12222} \eta_{2}^{4}+\frac{1}{4} C_{11122} \eta_{1}^{2} \eta_{2}^{2}+\frac{1}{6} C_{11112} \eta_{1}^{3} \eta_{2}+\frac{1}{2} C_{1122} \eta_{1} \eta_{2}^{2} \\
& +\frac{1}{12}\left(C_{11111}+3 C_{11112}+2 C_{11122}-3 C_{12222}-C_{22222}\right) \eta_{1} \eta_{2}^{3} \\
\Sigma_{2} & =C_{12} \eta_{1}+C_{11} \eta_{2}+\frac{1}{2} C_{112} \eta_{1}^{2}+\frac{1}{2} C_{222} \eta_{2}^{2}+\left(C_{111}+C_{112}-C_{222}\right) \eta_{1} \eta_{2} \\
& +\frac{1}{6} C_{1112} \eta_{1}^{3}+\frac{1}{6} C_{2222} \eta_{2}^{3}+\frac{1}{4}\left(C_{1111}+2 C_{1112}-C_{2222}\right) \eta_{1} \eta_{2}^{2} \\
& +\frac{1}{2} C_{1122} \eta_{1}^{2} \eta_{2}+\frac{1}{24} C_{22222} \eta_{2}^{4}+\frac{1}{24} C_{11112} \eta_{1}^{4}+\frac{1}{6} C_{11122} \eta_{1}^{3} \eta_{2}+\frac{1}{6} C_{12222} \eta_{1} \eta_{2}^{3} \\
& +\frac{1}{8}\left(C_{11111}+3 C_{11112}+2 C_{11122}-3 C_{12222}-C_{22222}\right) \eta_{1}^{2} \eta_{2}^{2}
\end{aligned}
$$


The ideal strength of materials is referred to the maximum value of the nominal stress in the plot of nominal stress versus nominal strain. Nominal stress and strain measures denoted, respectively, by $\sigma_{I}$ and $e_{I}$ in terms of the second P-K stress and Lagrangian strain are given as

$$
\begin{aligned}
& \sigma_{I}=\Sigma_{I} \sqrt{1+2 \eta_{I}}, \quad I=1,2, \\
& e_{I}=\sqrt{1+2 \eta_{I}}-1, \quad I=1,2 .
\end{aligned}
$$

It remains to obtain a set of data for $\Sigma_{I}$ versus $\eta_{I}$ with the aid of Eqs. (7a) and $(7 \mathrm{~b})$. To obtain $\Sigma_{1}$ versus $\eta_{1}$, we begin by giving a small increment to $\Sigma_{1}$ while keeping $\Sigma_{2}=0$. Subsequently, Eqs. (7a) and (7b) are solved for $\eta_{1}$ and $\eta_{2}$. In the next step, with $\Sigma_{2}=0$ we give an increment to the previous value of $\Sigma_{1}$ and calculate the corresponding new values of $\eta_{1}$ and $\eta_{2}$. This procedure is repeated until a sufficient number of data is obtained. $\Sigma_{2}$ versus $\eta_{2}$ is obtained in a similar manner.

\subsection{Approach based on ab-initio DFT calculations}

In first principles calculations, when a body is subjected to extension in a given direction then the other two perpendicular directions are kept fixed unless specified otherwise. Therefore, to model the uniaxial tension in the AC- (ZZ-) direction, the unit cell shown in Fig. 1 is strained along the $x_{1^{-}}\left(x_{2^{-}}\right)$direction in small increments and the deformation in $x_{2^{-}}\left(x_{1^{-}}\right)$ direction is adjusted in such a way that the corresponding stress along $x_{2^{-}}\left(x_{1^{-}}\right.$ ) direction vanishes after each increment. Computationally, in the current work, the relaxed condition is achieved by continuing the ionic iterations with the conjugate gradient method until the total energy of the system is minimized satisfactorily and the values of the stress along $x_{2^{-}}\left(x_{1^{-}}\right)$direction 
becomes less than $0.05 \mathrm{GPa}$. The algorithm for applying strain to the unit cell was previously described in Section 4.3.

\section{Results and discussion}

Throughout this section, the computational results for the morphological parameters, elastic constants, and ideal tensile strength of buckled stanene are presented. For the sake of examination of the buckling effect on the mechanical and morphological properties of stanene, the numerical results for planar stanene are also given. Graphene like stanene also belongs to 2D hexagonal lattice of the crystal class $D_{6 h}$. These 2D materials display clear prospects for application as flexible electronics, and thus comparison of their electro-mechanical properties are of interest. In the current work, their higher order elastic constants and ideal strengths are computed and compared. As mentioned earlier, these constants are computed based on two viewpoints of stress-strain relation and energy consideration. To gain confidence in the current calculations, the numerical values of the elastic constants and ideal strengths of graphene are compared to those of available experimental and computational results.

\subsection{Morphology of the free-standing stanene and its evolution during uniax- ial and biaxial loadings pertinent to $A C$ - and ZZ-directions}

The numerical values of equilibrium morphological parameters including lattice parameter and Sn-Sn bond length for both planar and buckled stanene as well as the buckling height and dihedral angle for the buckled stanene are calculated and given in this section. As mentioned previously, the morphology of stanene at equilibrium corresponds to the minimum total 


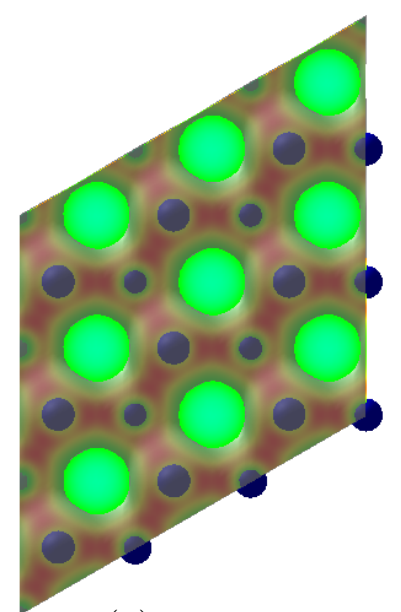

(a)

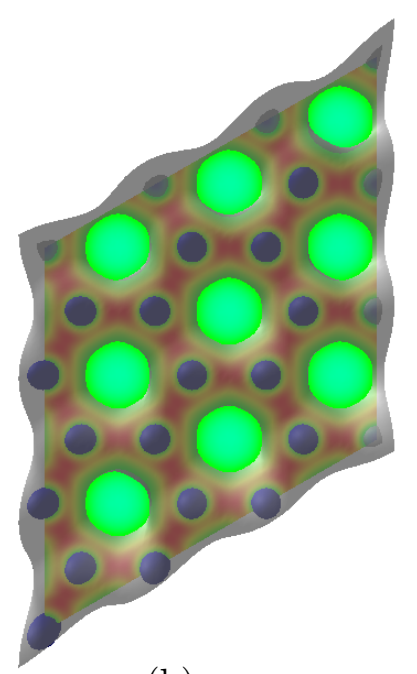

(b)

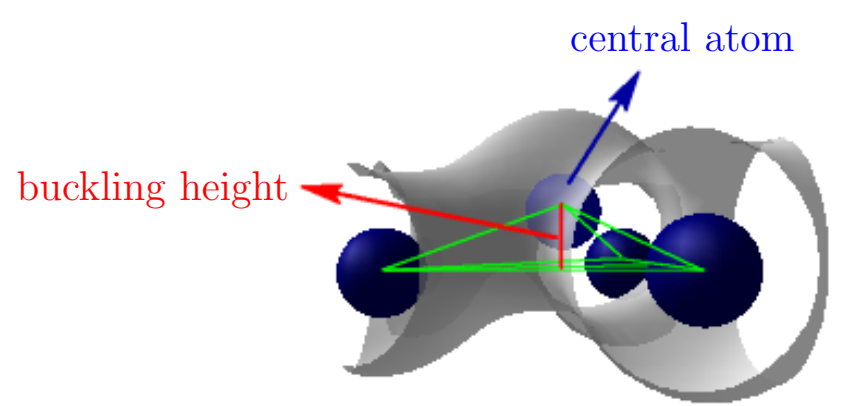

(c)

Figure 2: DFT calculations of (a) buckled equilibrium configuration, (b) planar equilibrium configuration, and (c) trigonal prism formed due to the $s p^{3}$ hybridized orbital in buckled configuration of stanene. 
energy of the unit cell. Moreover, at this configuration, the cohesive energy of the buckled stanene has been computed and compared with those of bulk as well as other monolayer structures. Our calculated results for morphological parameters and cohesive energy as well as those available in the literature are presented in Tables 3 and 4, respectively. Table 4 displays the calculated cohesive energies of stanene, tin, silicene, silicon, graphene, and carbon using VASP but with different potentials, local density approximation (LDA), projector augmented-wave (PAW), PAW-GGA, and PAWPerdew-Burke-Ernzerhof (PAW-PBE). In this table, our calculated data is also compared with some of the data given in the literature. Drummond et al. (2012) calculated the cohesive energy of silicene using DFT-LDA and DFT-PBE. Alfe et al. (2004) employed DFT-LDA to calculate this energy for silicon. Dunlap and Boettger (1996) used linear combination of Gaussiantype orbitals-fitting function (LCGTO-FF) in conjunction with both PerdewZunger (PZ) and Hedin-Lundqvist (HL) and calculated the cohesive energies of graphite and graphene. As it can be seen from Table 4, all the corresponding data fall within a reasonable range of each other..

It is observed that the agreement between the results are reasonable. The buckled and planar configurations obtained by DFT calculations are depicted in Figs. 2(a) and (b), respectively. The formation of dangling bonds on Sn atoms in the buckled configuration is recognized via the observation of a $s p^{3}$ hybridized orbital associated with the trigonal prism, Fig. 2(c). It is worthy to reiterate that for these obtained equilibrium parameters the components of the average stress associated with the unit cell vanish. In addition, the thickness of stanene can be estimated as $2 r_{v}+h_{b}$, where $r_{v}$ is the Van der 
Table 3: Comparison of the computed values of the morphological parameters including lattice parameter, Sn-Sn bond length, buckling height in units of $\AA$ dihedral angle in degree, and monolayer thickness in units of $\AA$ with the available results.

\begin{tabular}{llll}
\hline Morphological parameter & planar stanene & buckled stanene & \\
\hline Lattice parameter & 4.79 & 4.67 & (current study) \\
& 4.8 & 4.8 & (Manjanath et al., 2014) \\
& & 4.65 & (Xu et al., 2013) \\
Sn-Sn bond length & 2.766 & 2.828 & (Van den Broek et al., 2014) \\
& 2.77 & 2.88 & (current study) \\
Buckling height & & 2.82 & (Manjanath et al., 2014) \\
& - & 0.787 & (Van den Broek et al., 2014) \\
& & 0.8 & (current study) \\
Dihedral angle & - & 0.92 & (Manjanath et al., 2014) \\
Monolayer thickness & 4.79 & $51.45^{\circ}$ & (Van den Broek et al., 2014) \\
& & 5.457 & (current study) \\
\hline
\end{tabular}

Table 4: Comparison of the cohesive energies (eV/atom) of several monolayers with those of their corresponding bulks.

\begin{tabular}{|c|c|c|c|c|c|c|c|c|}
\hline & & stanene & $\operatorname{tin}$ & silicene & silicon & graphite & graphene & carbon \\
\hline \multirow[t]{4}{*}{ current study } & LDA & 3.44 & 3.85 & 4.64 & 5.14 & - & 8.88 & 8.91 \\
\hline & PAW & 3.43 & 3.87 & 4.63 & 5.34 & - & 8.98 & 9.00 \\
\hline & PAW-GGA & 2.82 & 3.74 & 3.92 & 4.67 & - & 8.91 & 7.91 \\
\hline & PAW-PBE & 2.76 & 3.05 & 3.90 & 4.61 & - & 8.00 & 7.85 \\
\hline Drummond et & DFT-LDA & & & 5.00 & & & & \\
\hline al. (2012) & DFT-PBE & & & 4.57 & & & & \\
\hline Alfe et al. (2004) & DFT-LDA & & & & 5.34 & & & \\
\hline Dunlap and & LCGTO-FF & & & & & 9.04 & 8.96 & \\
\hline \multirow[t]{3}{*}{ Boettger (1996) } & $(\mathrm{PZ})$ & & & & & & & \\
\hline & LCGTO-FF & & & & & 8.89 & 8.80 & \\
\hline & (HL) & & & & & & & \\
\hline
\end{tabular}

Waals radius and $h_{b}$ is the buckling height. According to this assumption, the thickness is calculated to be about $5.457 \AA$.

By applying three modes of strain loadings, namely uniaxial and biaxial 


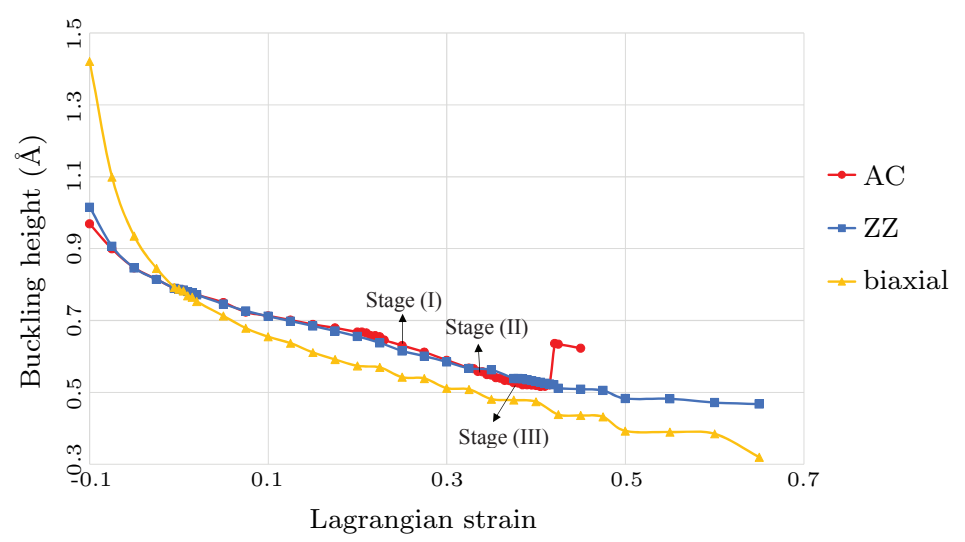

(a) Buckling height

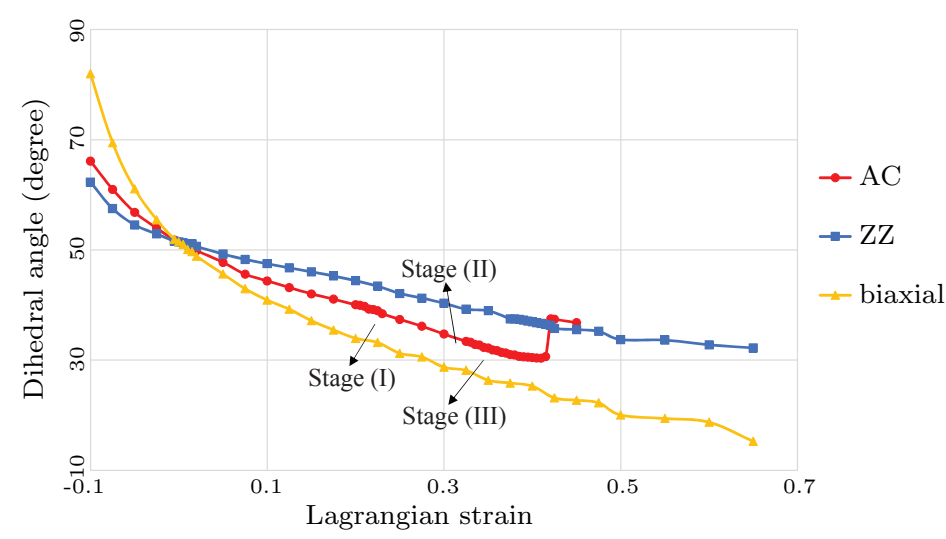

(b) Dihedral angle

Figure 3: (a) Buckling height and (b) dihedral angle versus Lagrangian strain in uniaxial $\mathrm{AC}, \mathrm{ZZ}$, and biaxial strain loadings.

loadings pertinent to AC- and ZZ-directions to the stanene unit cell as mentioned in Table 1, the changes in the buckling height and dihedral angle are examined. The variations of the buckling height $(\AA)$ and the dihedral angle (degree) with Lagrangian strain are shown in Figs. 3(a) and (b), respectively. Each of these figures contains three curves corresponding to the mentioned three modes of Lagrangian strain loadings. As noted earlier $\eta_{1} \neq 0, \eta_{2}=0$ and $\eta_{1}=0, \eta_{2} \neq 0$ indicate Lagrangian strain components associated with 


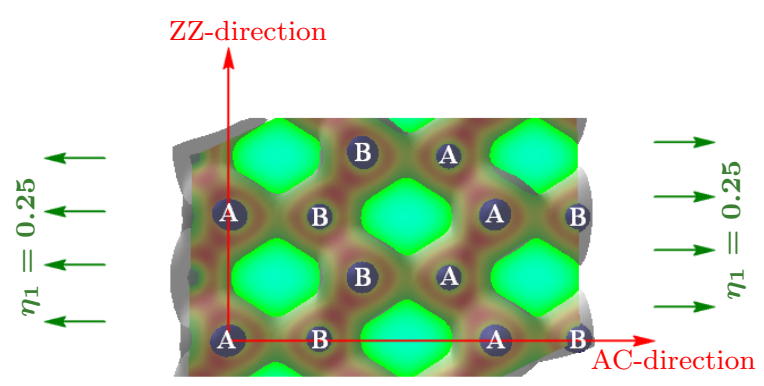

(a) Stage (I)

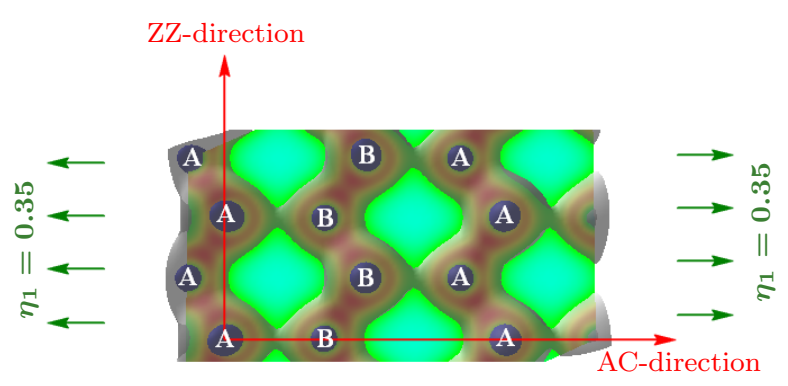

(b) Stage (II)

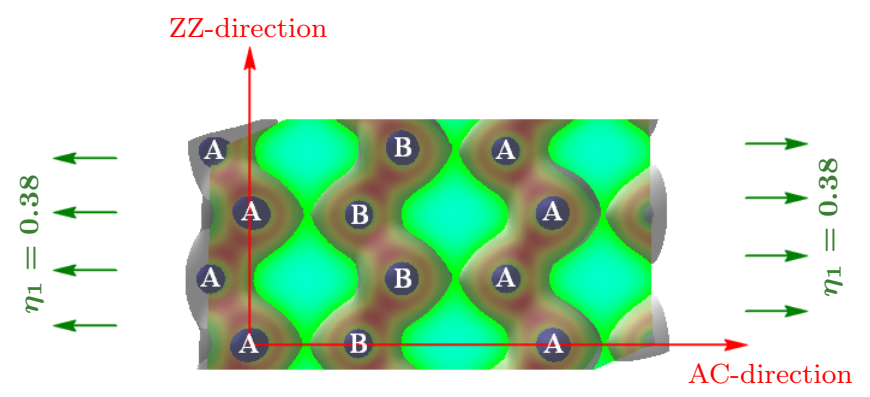

(c) Stage (III)

Figure 4: (a)-(c) The electronic charge distribution pertinent to the stages (I)-(III) indicated in Fig.3.

the uniaxial strain loading along AC- and ZZ-directions, respectively. The biaxial mode of loading corresponding to these directions is achieved by applying $\eta_{1}=\eta_{2} \neq 0$. As expected, both the buckling height and the dihedral angle keep decreasing as the applied strain increases for all the three modes, with the exception of an internal instability pertinent to the uniaxial strain loading in AC-direction occurring at the strain level of $\eta_{1} \approx 0.42$.

The trend of the evolution of the electronic charge distribution associated with the uniaxial extension along the AC-direction can be seen from Figs. 4(a)-(c), where the electronic charge distribution pertinent to the stages (I), (II), and (III) indicated in Figs. 3(a) and (b) are compared. From Figs. 4(a)-(c) it is observed that the progressively increasing extension along the AC-direction accompanies with the necking of the atomic bonds between 


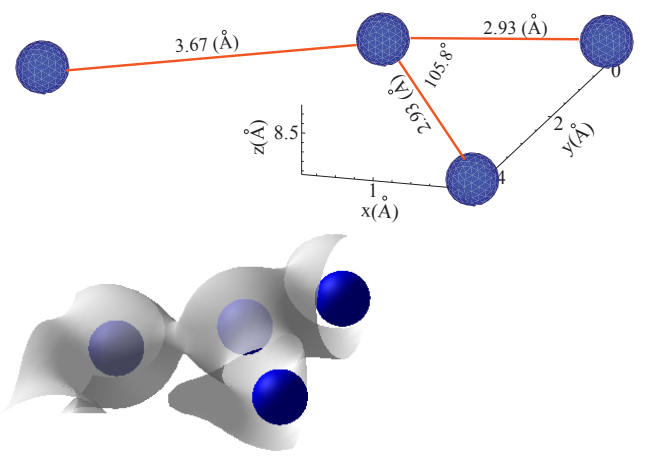

(a)

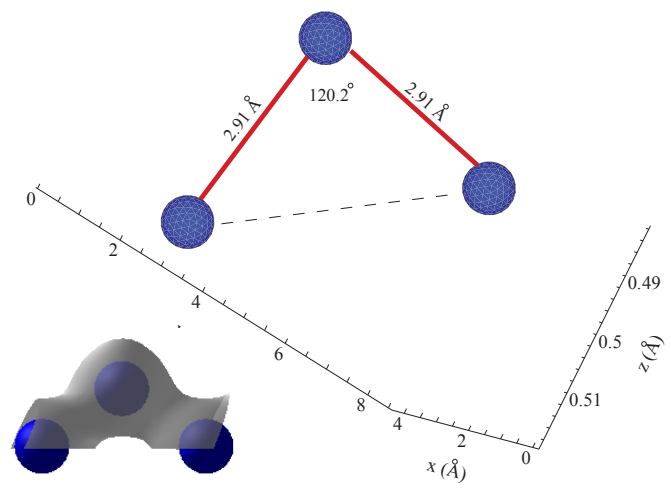

(b)

Figure 5: Structural geometries of the atomic bond angle pertinent to the buckled configuration of stanene under uniaxial extension along AC-direction of (a) $\eta_{1}=0.35$ and (b) $\eta_{1}=0.38$. The corresponding electronic charge distributions are also shown.

atoms $\mathrm{A}$ and $\mathrm{B}$; the projection of these atomic bonds onto the $\mathrm{AC}-\mathrm{ZZ}$ plane are parallel to the applied extension. From Fig. 4(a) which corresponds to $\eta_{1}=0.25$ it is seen that the formation of the necking has begun. A clear necking of the bonds has occurred at $\eta_{1}=0.35$ which is the threshold of the breakage of these atomic bonds, Fig. 4(b). Consequently, the $s p^{3}$ hybridized orbitals transform to $s p^{2}$ hybridized orbitals by further increase of the strain loading beyond $\eta_{1}=0.35$. At the Stage (III) corresponding to $\eta_{1}=0.38$, the transformation has taken place. For a closer scrutinization of this phenomenon, the structural geometries of the atomic bond angle pertinent to the loadings $\eta_{1}=0.35$ and $\eta_{1}=0.38$ are displayed in Figs. 5(a) and (b), respectively. These figures are accompanied with the corresponding electronic charge distribution. From Fig. 5(a), it is evident that the length of the necked Sn-Sn bond is $3.67 \AA$ which is much larger than the equilibrium bond length of $2.77 \AA$. This bond is in fact on the verge of rupture. Consequently, it is absent in the next displayed loading stage of $\eta_{1}=0.38$, Fig. 5(b). From Fig. 5(a), it is also seen that the bond angles between the overly extended bond and the two shorter bonds have distorted considerably away from $109.5^{\circ}$, the bond angle for the regular $s p^{3}$ hybridized orbital in the 

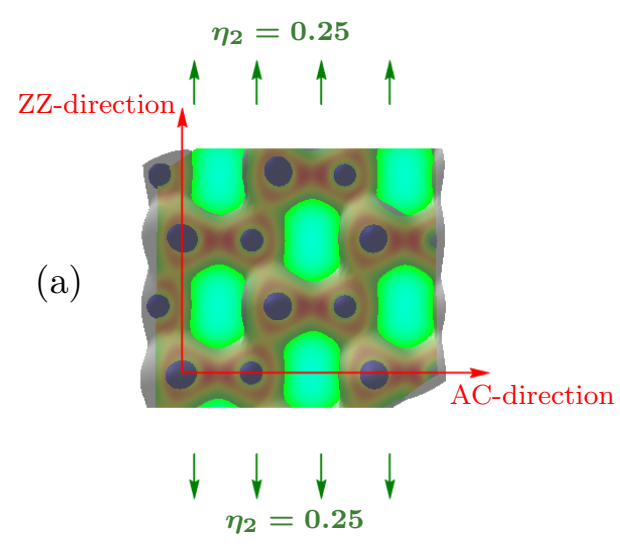
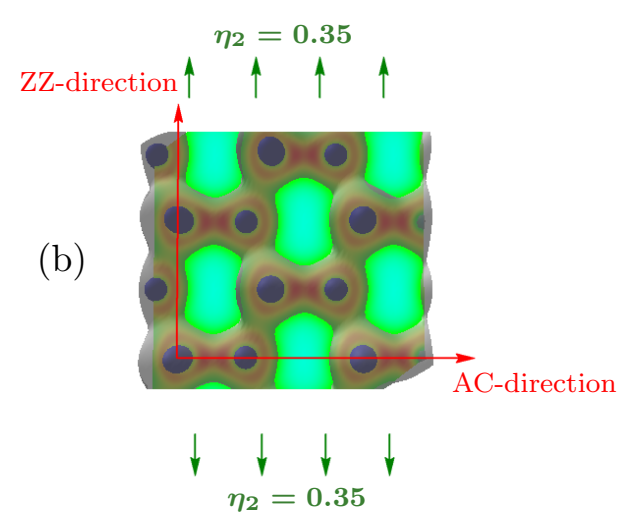

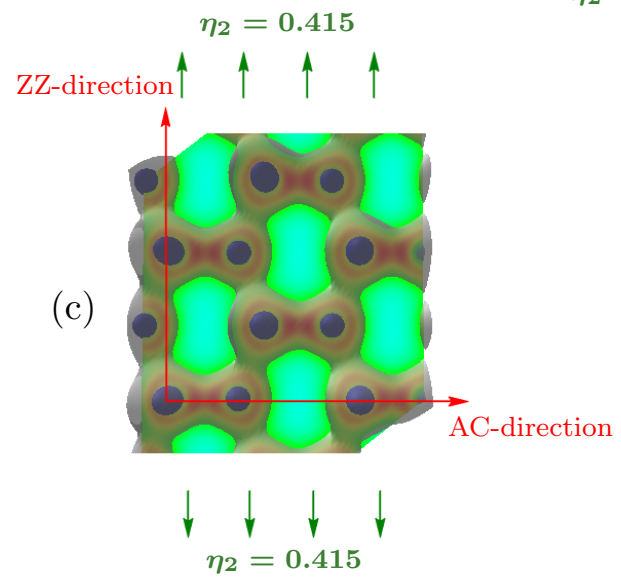

Figure 6: (a)-(c) The buckled configuration of stanene under uniaxial extension along the ZZ-direction for different values of the Lagrangian strain.

absence of any loadings. However, the bond angle between the two shorter bonds is about $105.8^{\circ}$ which is slightly different from $109.5^{\circ}$. At the strain level $\eta_{1}=0.38$ where the previously necked bond has broken, the bond angle between the shorter bonds becomes about $120.2^{\circ}$ which is equal to the bond angle in the $s p^{2}$ hybridized orbital in the absence of any loadings.

Likewise, the trend of the evolution of the electronic charge distribution of the buckled stanene with increasing of the applied uniaxial extension $\left(\eta_{2}=0.25,0.35,0.415\right)$ along the ZZ-direction is monitored in Figs. 6(a)-(c). It is seen that the hexagons become extended along the ZZ-direction with increasing the strain level. Also, thinning of the bonds whose projections on to the AC-ZZ plane are not perpendicular to the ZZ-direction is observed. 


\subsection{Fourth through tenth order elastic moduli tensors of stanene}

In this subsection, the fourth through tenth order elastic moduli tensors (i.e., the second through fifth order in Voigt notation) of stanene are evaluated according to both energy versus strain (E-S) and stress versus strain (S-S) considerations described in Section 4. As it was alluded to, due to the introduction of inaccuracy caused by post-processing needed to calculate Cauchy stresses from the energy computed by VASP, it is expected that E-S approach yields higher accuracy than S-S approach. In the energetic approach, strain energy density is calculated at several Lagrangian strain levels in each of the five different modes, which are given along with their corresponding simplified constitutive relations in Table 2, by using DFT calculations. For each mode of loading the obtained strain energy density $(\mathrm{N} / \mathrm{m})$ at different strain levels for two cases of planar and buckled stanene are plotted, respectively, in Figs. 7(a) and (b). Additionally, the strain energy density corresponding to graphene is also calculated using DFT and given in terms of the applied strain in Fig. 7(c) for each specified mode. By incorporating least square (LS) method the constitutive equation associated with each mode is fitted to the corresponding calculated DFT data, and subsequently all fourteen non-zero components of the elastic moduli tensors are calculated. In S-S approach, however, the components of Cauchy stress obtained directly by VASP are converted to the second P-K stress components using Eq. (3). Then, the second P-K stress is plotted in terms of the discrete values of the applied Lagrangian strain for the planar and buckled stanene as shown in Figs. 8(a) and (b), respectively. Using the same framework, the second P-K stress versus Lagrangian strain for graphene is also obtained and presented 
(a)

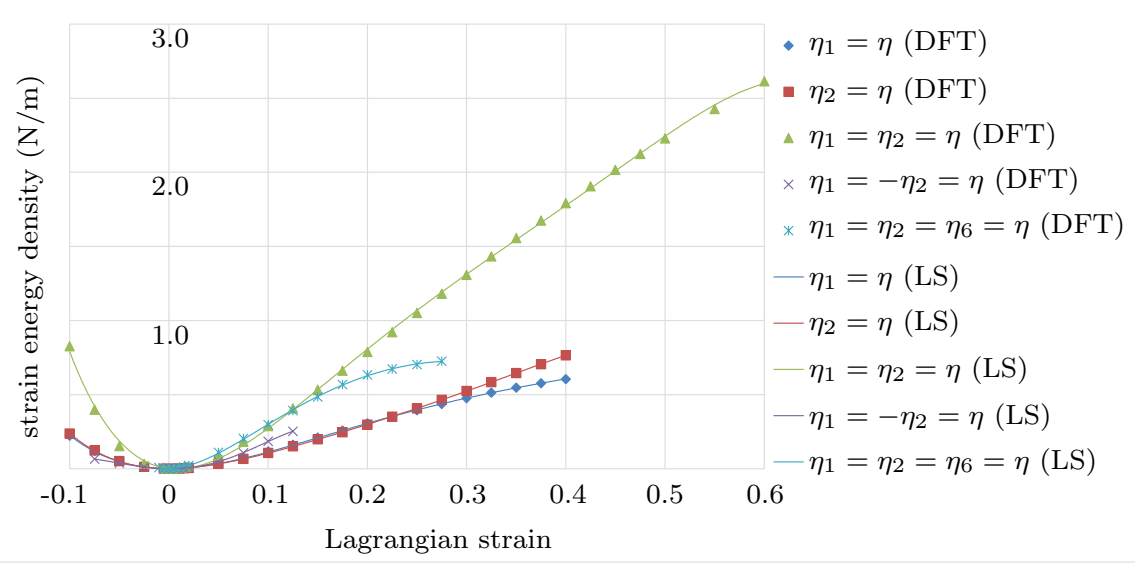

(b)

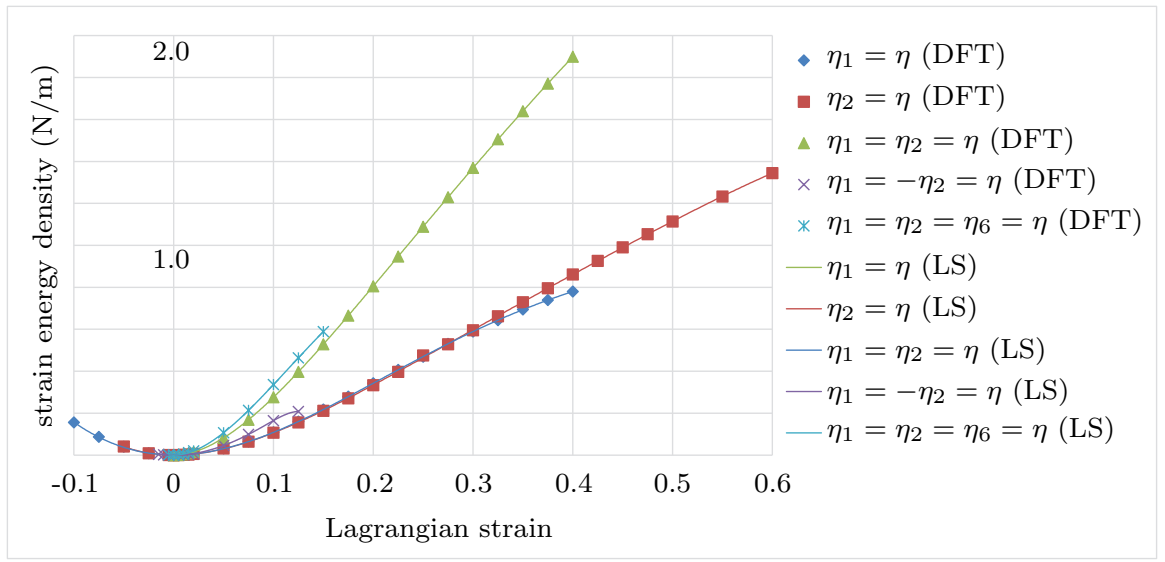

(c)

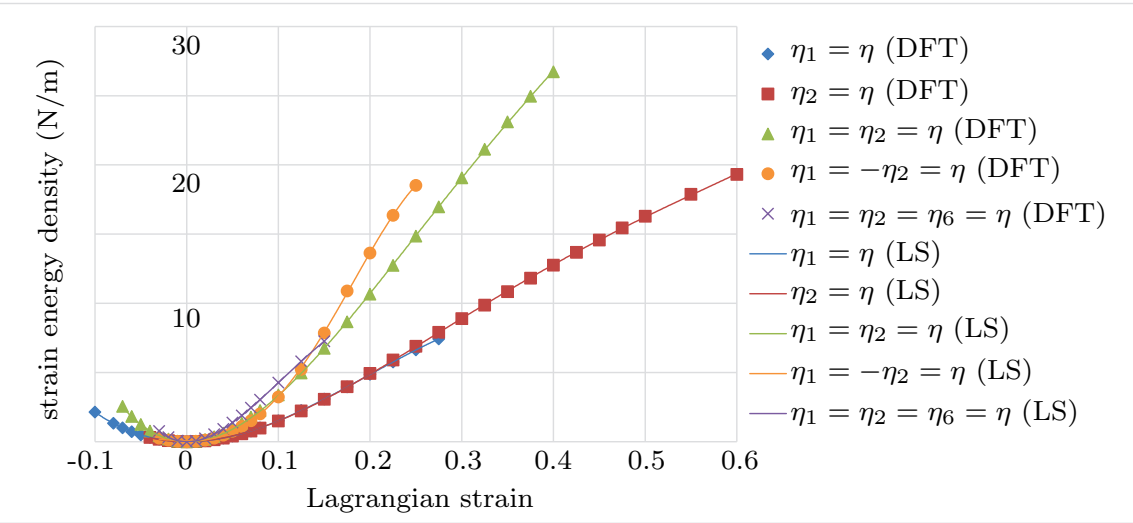

Figure 7: Strain energy density versus Lagrangian strain for (a) planar stanene, (b) buckled stanene, and (c) graphene, calculated using DFT for five different modes of loadings explained in Table 2. The simplified constitutive model for each mode is fitted (solid lines) to the corresponding data obtained from DFT. 
(a)

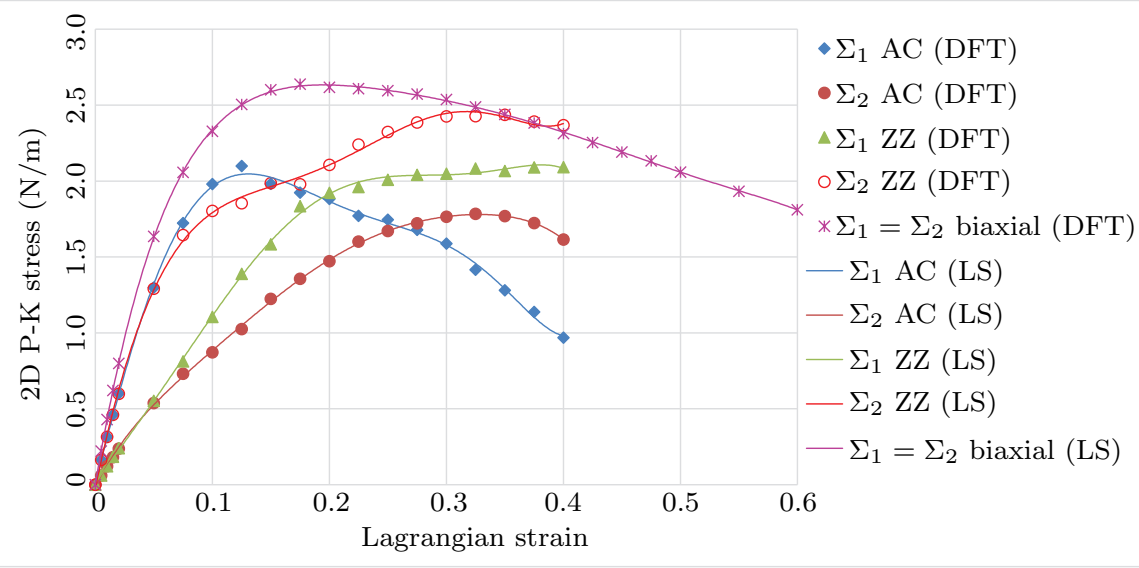

(b)

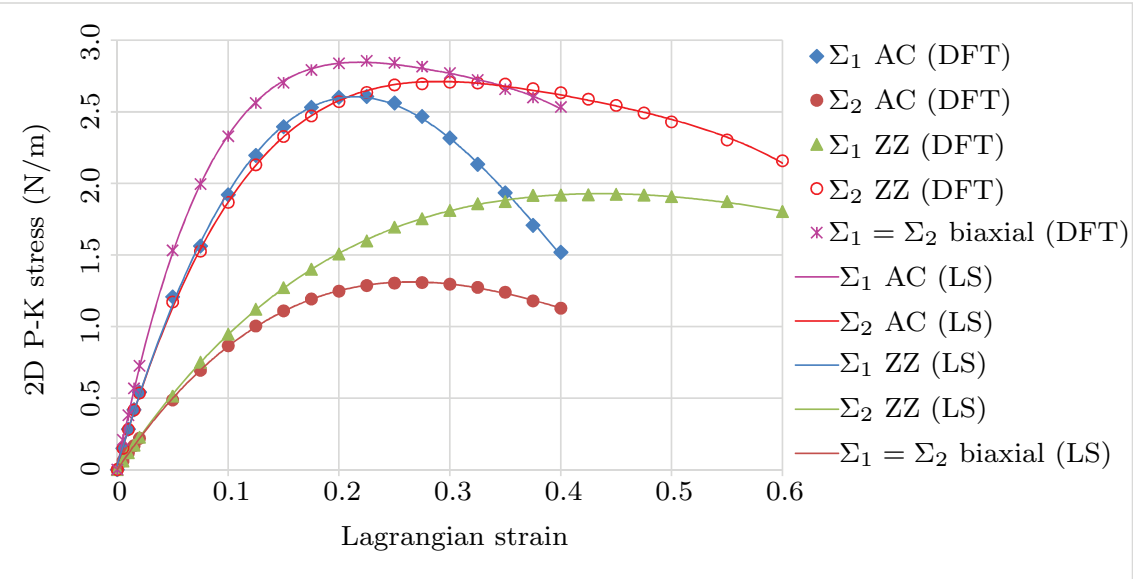

(c)

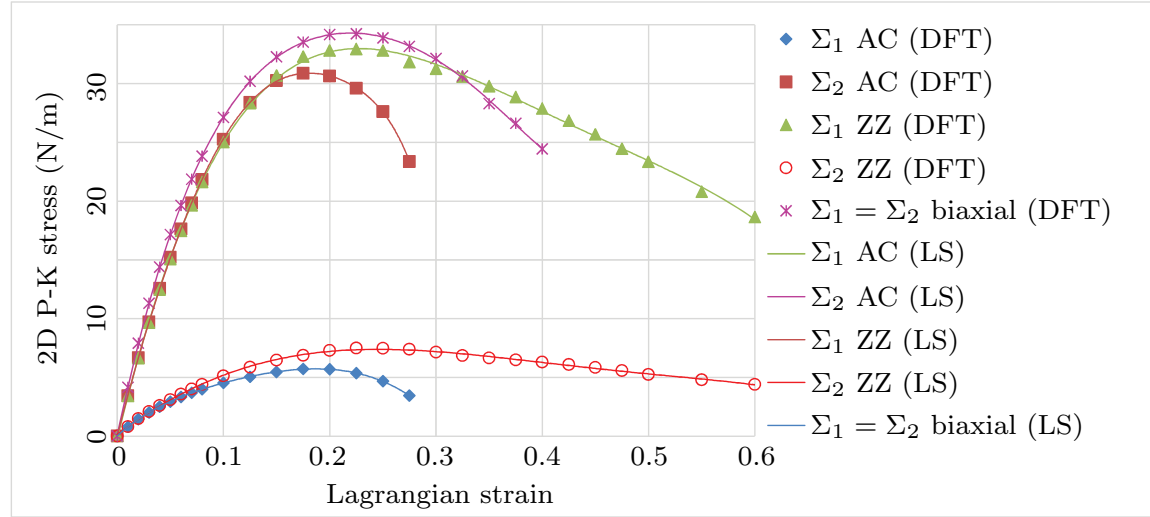

Figure 8: 2D P-K stress versus Lagrangian strain for (a) planar stanene, (b) buckled stanene, and (c) graphene, calculated using DFT for three different modes of loadings explained in Table 1. The simplified constitutive model for each mode is fitted (solid lines) to the corresponding data obtained from DFT. 
Table 5: Non-zero components of elastic moduli tensors $(\mathrm{N} / \mathrm{m})$ of order two through five for planar and buckled stanene incorporating stress-strain (S-S) and energy-strain (E-S) approaches.

\begin{tabular}{|c|c|c|c|c|}
\hline \multirow[t]{2}{*}{ Elastic constant } & \multicolumn{2}{|c|}{ Planar stanene } & \multicolumn{2}{|c|}{ Buckled stanene } \\
\hline & $(\mathrm{S}-\mathrm{S})$ & (E-S) & $(\mathrm{S}-\mathrm{S})$ & (E-S) \\
\hline$C_{11}$ & 31.6 & 32.0 & 27.6 & 26.6 \\
\hline$C_{12}$ & 15.8 & 17.2 & 11.5 & 10.6 \\
\hline$C_{111}$ & -316.1 & -348.2 & -145.6 & -148.2 \\
\hline$C_{112}$ & -155.6 & -149.2 & -105.2 & -91.5 \\
\hline$C_{222}$ & -379.3 & -394.5 & -163.4 & -213.0 \\
\hline$C_{1111}$ & 1692.8 & 2133.8 & 62.6 & 59.4 \\
\hline$C_{1112}$ & 1591.7 & 2125.5 & 168.9 & 565.8 \\
\hline$C_{1122}$ & 591.3 & 256.8 & 798.1 & 160.9 \\
\hline$C_{2222}$ & 3940.2 & 3526.8 & 1038.3 & 1076.6 \\
\hline$C_{11111}$ & -4112.2 & -6189.8 & 1735.0 & 2029.8 \\
\hline$C_{11112}$ & -8352.5 & -347.0 & -197.2 & -1764.4 \\
\hline$C_{11122}$ & 1017.2 & 3935.4 & -1803.0 & -4894.3 \\
\hline$C_{12222}$ & -1776.7 & 32994.7 & -101.2 & -9457.6 \\
\hline$C_{22222}$ & -12286.1 & -15410.4 & -2611.4 & -2788.2 \\
\hline
\end{tabular}

in Fig. 8 (c). In S-S approach, in order to recover all fourteen non-zero elastic constants, three modes of loadings are required. The modes of loadings and the pertinent simplified analytical expressions for the second P-K stress components in terms of the Lagrangian strains are given in Table 1. By incorporation of LS method these analytical expressions are fitted to the corresponding data of the second P-K stresses versus Lagrangian strains obtained from the first principles calculations, and subsequently the values of the elastic constants are determined. The non-zero components of the elastic 


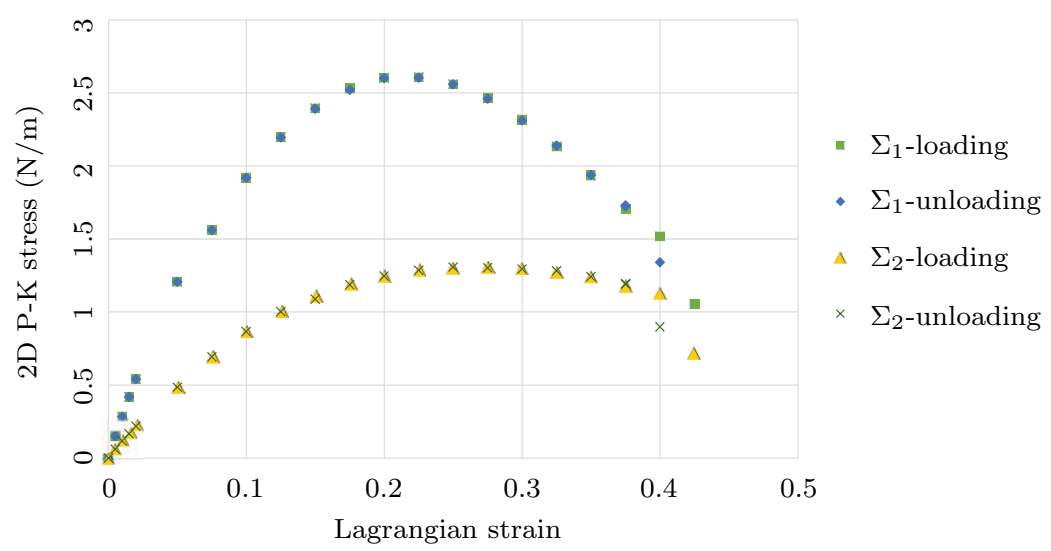

Figure 9: 2D P-K stress versus Lagrangian strain for buckled stanene subjected uniaxial strain (loading/unloading) along the AC-direction.

moduli tensors calculated for two cases of planar and buckled stanene via S-S and E-S approaches are listed in Table 5. Likewise, the elastic constants of graphene computed by the incorporation of both approaches are given in Table 6. The DFT results obtained by Wei et al. (2009) using S-S approach are also displayed in Table 6. Lee et al. (2008) have evaluated $C_{11}=340 \pm 50$ $\mathrm{N} / \mathrm{m}$ and $C_{111}=-690 \pm 120 \mathrm{~N} / \mathrm{m}$ for graphene using nanoindentation in an atomic force microscope which are within the range of the corresponding values reported in Table 6.

As explained in the previous section, the present large deformation continuum model is applicable as long as the response remains elastic. For elasticity the unloading and loading curves must coincide. Our analysis shows that the loading and unloading curves in the stress-strain diagram pertinent to the buckled stanene under uniaxial extension along the AC-direction coincide exactly up to the Lagrangian strain of about 0.4 . This observation is in accord with the sudden increase in the corresponding dihedral angle and buckling height depicted in Fig. 3. The noted loading and unloading behavior for the mentioned case is demonstrated in Fig. 9.

The goodness of the fifth order fit can be examined by calculating the root-mean square (rms) deviation defined as $\sqrt{\mathrm{SSE} / n}$, where SSE is the sum 
Table 6: Non-zero components of elastic moduli tensors $(\mathrm{N} / \mathrm{m})$ of order two through five for graphene incorporating stress-strain (S-S) and energy (E-S) approaches. The results reported by Wei et al. (2009) are obtained using DFT calculations and S-S consideration.

\begin{tabular}{llll}
\hline $\begin{array}{l}\text { Elastic } \\
\text { constant }\end{array}$ & $\begin{array}{l}\text { Graphene (current study) } \\
(\mathrm{S}-\mathrm{S})\end{array}$ & $\begin{array}{l}\text { Graphene (current study) } \\
(\mathrm{E}-\mathrm{S})\end{array}$ & $\begin{array}{l}\text { Graphene (Wei et al., 2009) } \\
(\mathrm{S}-\mathrm{S})\end{array}$ \\
\hline$C_{11}$ & 362.8 & 363.2 & 358.1 \\
$C_{12}$ & 69.3 & 71.8 & 60.4 \\
$C_{111}$ & -2439.8 & -1804.3 & -2693.3 \\
$C_{112}$ & -532.8 & -732.4 & -460.8 \\
$C_{222}$ & -2567.9 & -2508.7 & -2817.0 \\
$C_{1111}$ & 7227.0 & 1557.2 & 10358.9 \\
$C_{1112}$ & 2323.4 & 2372.4 & 2287.6 \\
$C_{1122}$ & 4587.8 & 3858.9 & 2582.8 \\
$C_{2222}$ & 6973.3 & 9787.0 & 13416.2 \\
$C_{11111}$ & -37574.4 & -30632.4 & -33446.7 \\
$C_{11112}$ & -13100.2 & -7746.0 & -13046.6 \\
$C_{11122}$ & 7974.6 & -44408.6 & 7508.2 \\
$C_{12222}$ & -702.9 & -100943.1 & -88.4 \\
$C_{2222}$ & -39465.6 & -19237.2 & -31383.8 \\
\hline
\end{tabular}

of error squares and $n$ is the number of the data points used in the fit. The rms deviation for the fifth order approximation is computed to be 0.0415 $\mathrm{N} / \mathrm{m}$ for the planar stanene with 88 data points of stress-strain and 0.055 $\mathrm{N} / \mathrm{m}$ for the buckled stanene with 126 data points.

\subsection{Ideal strength}

The methodologies for the calculation of the theoretical or ideal strength in the AC- and ZZ-directions was explained in Section 5. The ideal strength corresponds to the maximum value in the plot of the nominal or engineering stress versus nominal or engineering strain. In summary, in one method using the DFT result first we calculate the components of the elastic moduli tensors by incorporating E-S or S-S approach. Utilizing these elastic constants in conjunction with the enhanced continuum-based constitutive 
relation discussed earlier, nominal stress versus nominal strain is plotted, and subsequently the corresponding ideal strength is obtained. The other method is more direct, in that the nominal stress is directly obtained from DFT results, without incorporating the elastic constants and the continuum model.

The elastic response of buckled stanene subjected to uniaxial tensile stress (strain-controlled) in the AC- and ZZ-directions in terms of nominal stress $(\mathrm{N} / \mathrm{m})$ versus nominal strain are shown in Figs. 10 (a) and (b), respectively. The results of the continuum model via S-S and E-S approaches as well as the result obtained directly from DFT are compared. It is seen that the result from E-S approach is in better correspondence with DFT result than S-S approach. For the sake of comparison graphs of nominal stress-strain for planar stanene corresponding to the uniaxial tensile stress in the AC- and ZZ-directions are shown in Figs. 10 (c) and (d), respectively. Furthermore, the elastic response for $\mathrm{AC}$ and $\mathrm{ZZ}$ under uniaxial tensile stress pertinent to graphene are shown in Figs. 11 (a) and (b), respectively. Examination of the behaviors of buckled stanene (Figs. 10 (a) and (b)) with those of graphene (Figs. 11 (a) and (b)) reveals that the theoretical strength of graphene is roughly ten times that of buckled stanene and, moreover, the stiffness of graphene in response to carrying uniaxial tensile stress is about ten times larger.

Quantitative values of the ideal strengths $(\mathrm{N} / \mathrm{m})$ for planar and buckled stanene along $\mathrm{AC}\left(\sigma_{1}^{t}\right)$ and $\mathrm{ZZ}\left(\sigma_{2}^{t}\right)$ directions are given in Table 7 . The corresponding values of the nominal strains, $e_{1}^{t}$ and $e_{2}^{t}$ are also provided in the Table. Stanene in its natural buckled state exhibits large ideal strength 


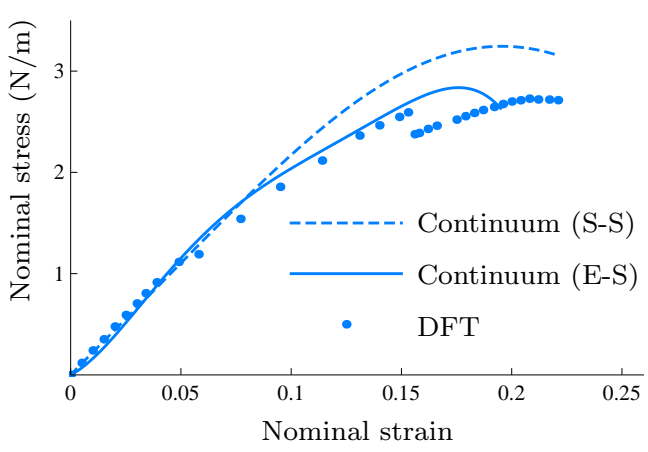

(a) Buckled stanene (AC-direction)

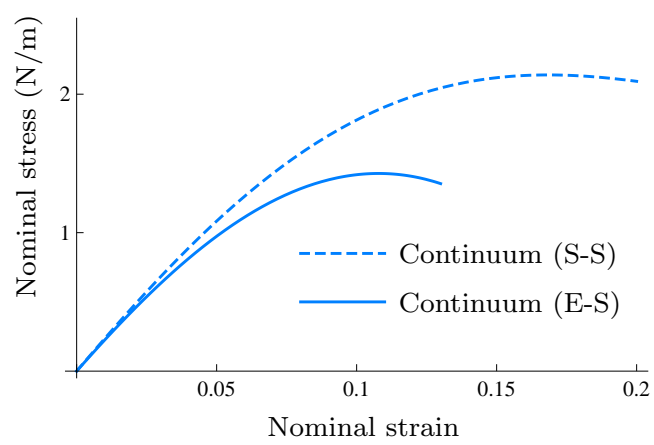

(c) Planar stanene (AC-direction)

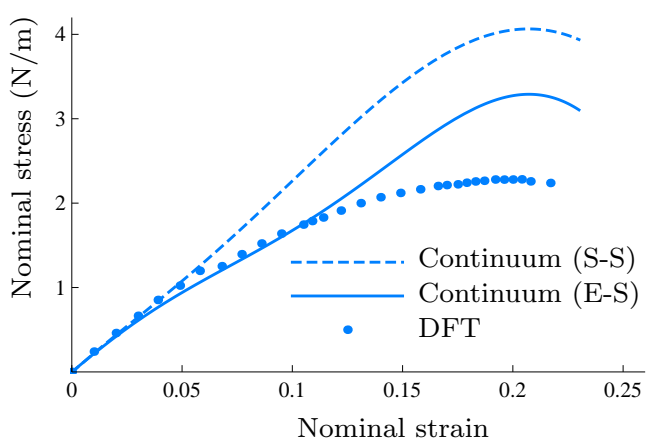

(b) Buckled stanene (ZZ-direction)

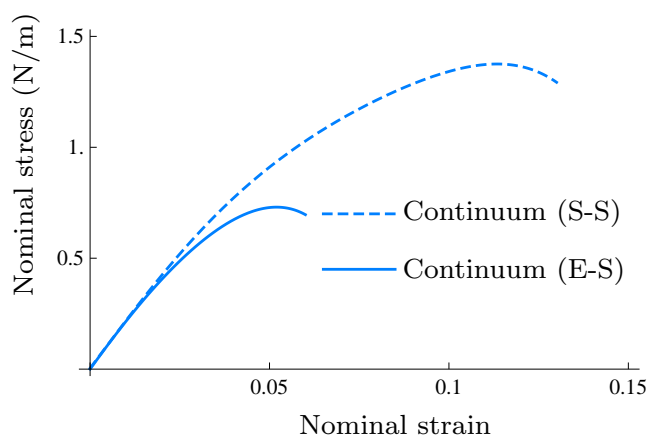

(d) Planar stanene (ZZ-direction)

Figure 10: The stress-strain curves for the uniaxial tensile stress (strain-controlled) for (a), (b) buckled stanene and (c), (d) planar stanene. (a) and (c) correspond to the tension in AC-direction, whereas (b) and (d) correspond to ZZ-direction. In parts (a) and (b), the symbols represent the results of DFT calculations. The solid and dashed lines are obtained based on the continuum model with the incorporation of the elastic moduli tensors obtained using DFT calculations together with E-S approach (solid line) and S-S approach (dashed line).

than the planar form. For the buckled stanene it is seen that the continuum results calculated via E-S approach are in better agreement with the DFT results than those via S-S approach. The ideal strengths computed in the current work are within reasonable agreement of those reported in the literature by Wei et al. (2009) and Delfani and Shodja (2013). It is noteworthy to mention that the ideal strengths reported by Delfani and Shodja (2013) 


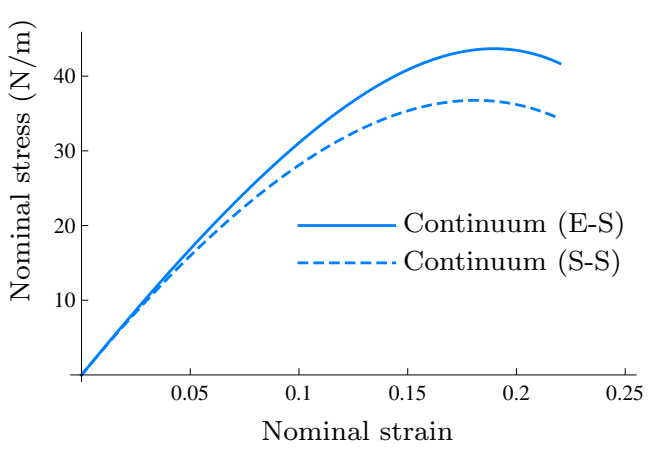

(a) Graphene (AC-direction)

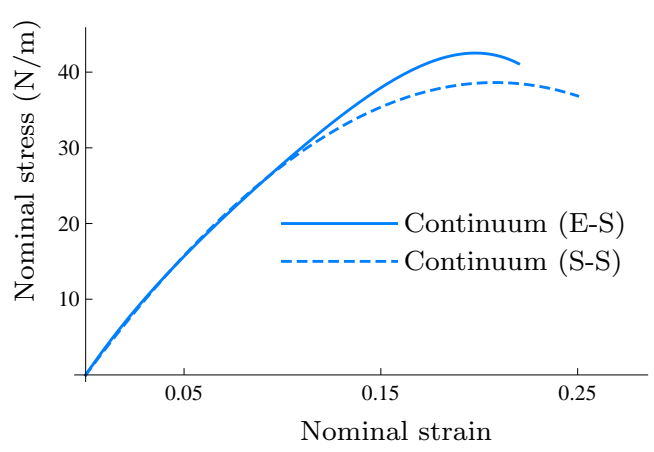

(b) Graphene (ZZ-direction)

Figure 11: The stress-strain curves for the uniaxial tensile stress (strain-controlled) for graphene. (a) and (b) correspond to the tension in AC- and ZZ-directions, respectively. The solid and dashed lines are obtained based on the continuum model with the incorporation of the elastic moduli tensors obtained using DFT calculations together with E-S approach (solid line) and S-S approach (dashed line).

were obtained analytically as the limiting cases of the corresponding carbon nanotubes (CNTs) as their radii $\rightarrow \infty$. They considered tubes with different chiralities and, therefore, their theory applies to any arbitrary orientations rather than just AC- or ZZ-directions. Lee et al. (2008) employed nanoindentation in an atomic force microscope and found the breaking strength of graphene to be $42 \pm 4 \mathrm{~N} / \mathrm{m}$ which is in excellent agreement with the current result obtained from the energetic approach (E-S).

\subsection{Young's modulus}

Young's moduli of stanene along the AC- and ZZ-directions, $E_{1}^{A C}$ and $E_{2}^{Z Z}$ are the initial slope of $\sigma_{I}$ versus $e_{I}, I=1,2$, respectively. That is

$$
\begin{aligned}
& E_{1}^{A C}=\left.\frac{d \sigma_{1}}{d e_{1}}\right|_{e_{1}=0}, \\
& E_{2}^{Z Z}=\left.\frac{d \sigma_{2}}{d e_{2}}\right|_{e_{2}=0},
\end{aligned}
$$


Table 7: Ideal strengths $(\mathrm{N} / \mathrm{m})$ of planar and buckled stanene along AC $\left(\sigma_{1}^{t}\right)$ and $\mathrm{ZZ}\left(\sigma_{2}^{t}\right)$ directions and comparison to those of graphene. The corresponding strains, $e_{1}^{t}$ and $e_{2}^{t}$ are also displayed.

\begin{tabular}{|c|c|c|c|c|c|c|c|c|c|}
\hline & \multicolumn{5}{|c|}{ Stanene (current study) } & \multicolumn{4}{|c|}{ Graphene } \\
\hline & \multirow{2}{*}{\multicolumn{2}{|c|}{ Planar }} & \multirow{2}{*}{\multicolumn{3}{|c|}{ Buckled }} & \multirow{2}{*}{\multicolumn{2}{|c|}{ current study }} & Wei et al. & Delfani and \\
\hline & & & & & & & & (2009) & Shodja (2013) \\
\hline & \multicolumn{2}{|c|}{ continuum } & \multicolumn{2}{|c|}{ continuum } & \multirow[b]{2}{*}{ DFT } & \multicolumn{2}{|c|}{ continuum } & continuum & continuum \\
\hline & $\mathrm{S}-\mathrm{S}$ & E-S & $\mathrm{S}-\mathrm{S}$ & E-S & & S-S & E-S & S-S & analytical \\
\hline$\sigma_{1}^{t}$ & 2.14 & 1.43 & 3.24 & 2.78 & 2.59 & 36.76 & 43.63 & 38.61 & 38.59 \\
\hline$e_{1}^{t}$ & 0.17 & 0.11 & 0.20 & 0.18 & 0.15 & 0.18 & 0.19 & 0.26 & 0.26 \\
\hline$\sigma_{2}^{t}$ & 1.37 & 0.73 & 4.05 & 3.29 & 2.28 & 38.61 & 42.52 & 34.82 & 34.79 \\
\hline$e_{2}^{t}$ & 0.11 & 0.05 & 0.21 & 0.21 & 0.20 & 0.21 & 0.20 & 0.19 & 0.19 \\
\hline
\end{tabular}

moreover, Poisson's ratios along the AC- and ZZ-directions, $\nu_{12}^{A C}$ and $\nu_{21}^{Z Z}$ are defined as

$$
\begin{gathered}
\nu_{12}^{A C}=-\left.\frac{d e_{2}}{d e_{1}}\right|_{e_{1}=0}, \\
\nu_{21}^{Z Z}=-\left.\frac{d e_{1}}{d e_{2}}\right|_{e_{2}=0} .
\end{gathered}
$$

Within infinitesimal deformations (i.e., in the neighborhood of $e_{I} \approx 0$ ) the role of higher order elastic moduli tensors is negligible. From Eq. (A.1a) given in Appendix A, the second order elastic moduli tensor of stanene is isotropic and, in particular, $C_{11}=C_{22}$. Thus, for general infinitesimal in-plane deformations induced by the uniaxial tensile stress applied in any direction, in-plane Young's modulus and Poisson's ratio are, respectively, 
defined as

$$
\begin{aligned}
& E=\frac{C_{11} C_{22}-C_{12}^{2}}{C_{22}}, \\
& \nu=\frac{C_{12}}{C_{22}} .
\end{aligned}
$$

The in-plane Young's moduli of planar and buckled stanene corresponding to AC- and ZZ-directions are, respectively, evaluated using Eqs. (9) and (10). These parameters can also be estimated via Eqs. (11). Estimates for Young's modulus and Poisson's ratio are summarized in Table 8. Young's modulus is in units of $\mathrm{N} / \mathrm{m}$. Dividing by the thickness of stanene it can be provided in units of $\mathrm{N} / \mathrm{m}^{2}$. A brief discussion on the estimation of the thicknesses of the planar stanene was given in Section 6.1, and the estimated values are listed in Table 3. The values of Young's modulus and Poisson's ratio from the literature are also reported in Table 8 for comparison. Van den Broek et al. (2014) have employed DFT calculations and reported Young's modulus in units of GPa without referring to the thickness used. Modarresi et al. (2014) have exploited molecular mechanics with stretching and bending potential terms and obtained the in-plane stiffness for stanene. In their work, the unknown parameters of the molecular mechanics have been evaluated using DFT calculations. It is observed that there is a reasonable agreement between the values of Young's modulus calculated in the current study and that of Van den Broek et al. (2014). In view of the fact that Modarresi et al. (2014) have employed molecular mechanics and for simplification they have disregarded the dihedral term in their potential, there is a relatively large discrepancy between the obtained values for Young's modulus. Moreover, in order to examine the effect of buckling on Young's modulus and Poisson's 
Table 8: Young's modulus and Poisson's ratio of buckled stanene.

\begin{tabular}{lll}
\hline & Young's Modulus & Poisson's ratio \\
\hline Eqs. (11a) and (11b), S-S approach & $22.81 \mathrm{~N} / \mathrm{m}(41.80 \mathrm{GPa})$ & 0.42 \\
Eqs. (11a) and (11b), E-S approach & $22.38 \mathrm{~N} / \mathrm{m}(41.01 \mathrm{GPa})$ & 0.40 \\
Eqs. (9a) and (10a) & $23.8 \mathrm{~N} / \mathrm{m}$ & 0.40 \\
Eqs. (9b) and (10b) & $24.2 \mathrm{~N} / \mathrm{m}$ & 0.40 \\
Van den Broek et al. (2014) & $46.3 \mathrm{GPa}$ & 0.62 \\
Modarresi et al. (2014) & $40 \mathrm{~N} / \mathrm{m}$ & \\
\hline
\end{tabular}

ratio, the corresponding values have also been calculated for planar stanene incorporating the stress approach

$$
E_{\text {planar }}=23.70 \mathrm{~N} / \mathrm{m}, \quad \nu_{\text {planar }}=0.50 .
$$

From the calculated values of theoretical strength and Young's modulus it isseen that $\sigma^{t} \approx 0.1 E$ which is a well known estimation for defect free materials. Moreover, employing the second order elastic constants given in Table 6, the in-plane Young's modulus and Poisson's ratio are also computed for graphene as displayed in Table 9. Comparison with the results of Table 8 for stanene reveals that $E_{\text {graphene }}$ is about fifteen times $E_{\text {stanene }}$, and $\nu_{\text {graphene }}$ is about half of $\nu_{\text {stanene }}$. 
Table 9: Young's modulus and Poisson's ratio of graphene.

\begin{tabular}{lll}
\hline & Young's Modulus (N/m) & Poisson's ratio \\
\hline Eqs. (11a) and (11b), S-S approach & 349.56 & 0.19 \\
Eqs. (11a) and (11b), E-S approach & 349.01 & 0.20 \\
Wei et al. (2009) & 348 & 0.169 \\
Cadelano et al. (2009) & 312 & 0.31 \\
Lee et al. (2008) & 342 & 0.165 \\
Kudin et al. (2001) & 345 & 0.149 \\
\hline
\end{tabular}

\section{Conclusion}

In pursuit of an accurate determination of the mechanical properties of planar and buckled stanene the large deformation continuum theory combined with the first-principles DFT has been utilized. Keeping in mind that stanene as well as graphene is a 2D hexagonal lattice of the crystal class, $D_{6 h}$ possessing a six-fold rotation axis perpendicular to its plane, incorporation of an appropriate higher order constitutive equation to capture the symmetries is inevitable. With the incorporation of the second order (in Voigt notation) elastic modulus tensor while neglecting the higher order ones, one can at best capture the isotropic response of stanene. While this assumption is reasonable within infinitesimal deformation theory, it degenerates within moderate through large deformations. It is seen that consideration of second through fifth order elastic modulus tensors can capture the six-fold symmetry of stanene belonging to $D_{6 h}$ class with high accuracy. To compute all 
fourteen independent elastic constants, five simple modes of strain loadings in the case of E-S approach and three simple modes of strain loadings in the case of S-S approach are proposed. After calculation of the elastic moduli tensors for stanene and graphene, their ideal strengths, Young's moduli, and Poisson's ratios are also obtained.

Furthermore, for different modes of strain loadings, i.e., uniaxial and biaxial loadings pertinent to $\mathrm{AC}$ - and ZZ-directions, the variations of the buckling height and dihedral angle as well as the trend of the evolution of the electronic charge density distribution are examined. Scrutinization of the electronic charge distribution reveals the phenomenon of the formation of necking in the Sn-Sn bonds when stanene is subjected to large uniaxial extension, $\eta_{1}$ along the AC-direction. At $\eta_{1}$ about 0.25 necking initiates, and the threshold of the breakage of these atomic bonds is at $\eta_{1}$ about 0.35 . At $\eta_{1}=0.35$ the bond length is about $3.67 \AA$, which is much larger than the equilibrium bond length of $2.77 \AA$. As a result, the $s p^{3}$ hybridized orbitals transform to $s p^{2}$ hybridized orbitals when the applied strain is increased beyond $\eta_{1}=0.35$. Further discussion on confirmation of the transformation is given through consideration of the structural geometries of the atomic bond angles pertinent to the loadings $\eta_{1}=0.35$ and 0.38 .

\section{Acknowledgement}

The authors acknowledge the support of Iran National Science Foundation(INSF)(Grant number 92021276). 


\section{References}

Alfe, D., Gillan, M.J., Towler, M.D., Needs, R.J., 2004. Diamond and $\beta$ tin structures of Si studied with quantum Monte Carlo calculations. Phys. Rev. B. Vol. 70, 214102.

Balendhran, S., Walia, S., Nili, H., Sriram, S., Bhaskaran, M., 2015. Elemental analogues of graphene: silicene, germanene, stanene, and phosphorene. Small. Vol. 11, 640-652.

Blochl, P.E., 1994. Projector augmented-wave method. Phys. Rev. B. Vol. 50(24), 17953.

Cadelano, E., Palla, P., Giordano, S., Colombo, L., 2009. Nonlinear elasticity of monolayer graphene. Phys. Rev. Lett. Vol. 102, 235502.

Dunlap, B.I. and Boettger, J.C., 1996. Local-density-functional study of the fullerenes, graphene and graphite. J. Phys.: At. Mol. Opt. Phys. Vol. 29, 4907-4913.

Delfani, M.R., Shodja, H.M., Ojaghnezhad, F., 2013. Mechanics and morphology of single-walled carbon nanotubes: from graphene to the elastica. Phil. Mag. Vol. 93(17), 2057-2088.

Delfani, M.R. and Shodja, H.M., 2013. An enhanced continuum modeling of the ideal strength and the angle of twist in tensile behavior of single-walled carbon nanotubes. J. App. Phys. Vol. 114, 053521.

Drummond, N.D., Zolyomi, V., FalKo, V.I., 2012. Electrically tunable band gap in silicene. Phys. Rev. B, Vol. 85(7), 075423. 
Fei, R., and Yang, L., 2014. Strain-Engineering the Anisotropic Electrical Conductance of Few-Layer Black Phosphorus. Nano Lett. Vol. 14, 28842889.

Fumi, F.G., 1952. Third-order elastic coefficients in trigonal and hexagonal crystals. Phys. Rev., Vol. 86(4), 561.

Gross, M., 2014. Stanene the next miracle material? Chemistry and Industry, Vol. 78(9), 24-27.

Hasan, M.Z., and Kane, C.L., 2010. Colloquium: Topological insulators. Rev. Mod. Phys. Vol. 82, 3045-3067.

Kresse, G., Hafner, J., 1994. ab-initio molecular-dynamics simulation of the liquid-metal amorphous-semiconductor transition in germanium. Phys. Rev. B. Vol. 49(20), 14251.

Kresse, G., Hafner, J., 1993. ab-initio molecular dynamics for liquid metals. Phys. Rev. B. Vol. 47(1), 558.

Kresse, G., Furthmuller, J., 1996. Efficient iterative schemes for ab-initio total-energy calculations using a plane-wave basis set. Phys. Rev. B, Vol. 54(16), 11169.

Kresse, G., Furthmuller, J., 1996. Efficiency of ab-initio total energy calculations for metals and semiconductors using a plane-wave basis set. Com. Mat. Sci., Vol. 6(1), 15-50.

Kudin, K.N., Scuseria, G., Yakobson, B., 2001. C 2 F, BN, and C nanoshell elasticity from ab initio computations. Phys. Rev. B. Vol. 64, 235406. 
Lee, Ch., Wei, X., Kysar, J.W., Hone, J., 2008. Measurement of the Elastic Properties and Intrinsic Strength of Monolayer Graphene. Science. Vol. $321,385-388$.

Liu, C.C., Jiang, H., Yao, Y., 2011. Low-energy effective Hamiltonian involving spin-orbit coupling in silicene and two-dimensional germanium and tin. Phys. Rev. B., Vol. 84, 195430.

Malvern, L.E., 1969. Introduction to the mechanics of a continuous media, Prentice-Hall, Inc. Englewood Cliffs, New Jersy.

Manjanath, A., Kumar, V., Singh, A.K., 2014. Mechanical and electronic properties of pristine and Ni-doped Si, Ge, and Sn sheets. Phys. Chem. Chem. Phys., Vol. 16, 1667-1671.

Min, H., Hill, J.E., Sinitsyn, N.A., Sahu, B.R., Kleinman, L., MacDonald, A.H., 2006. Intrinsic and Rashba spin-orbit interactions in graphene sheets. Phys. Rev. B. Vol. 74, 165310.

Modarresi, M., Kakoee, A., Mogulkoc, Y., Roknabadi, M.R. (2015). Effect of external strain on electronic structure of stanene. Computational Materials Science, 101, 164-167.

Monkhorst, H.J., Pack, J.D., 1976. Special points for Brillouin-zone integrations. Phys. Rev. B. Vol. 13(12), 5188.

Morris Jr, J.W., Krenn, C.R., 2000. The internal stability of an elastic solid. Phil. Mag. A, Vol. 80(12), 2827-2840 
Nye, J.F., 1985. Physical Properties of Crystals: Their Representation by Tensors and Matrices, Oxford Science Publications, Clarendon, Oxford.

Ojaghnezhad, F., Shodja, H.M., 2012. A combined first principles and analytical treatment for determination of the surface elastic constants: application to $\mathrm{Si}(001)$ ideal and reconstructed surfaces. Phil. Mag. Lett., Vol. 92(1), 7-19.

Peng, Q., Ji, W., De, S., 2012a. Mechanical properties of the hexagonal boron nitride monolayer: ab-initio study. Com. Mat. Sci. Vol. 56, 11-17.

Peng, Q., Liang, C., Ji, W., De, S., 2013. A first principles investigation of the mechanical properties of g-ZnO: the graphene-like hexagonal zinc oxide monolayer. Comp. Mat. Sci., Vol. 68, 320-324.

Perdew, J.P., Burke, K., Ernzerhof, M., 1996. Generalized gradient approximation made simple. Phys. Rev. Lett., Vol. 77(18), 3865.

Perdew, J.P., Ruzsinszky, A., Csonka, G.I., Vydrov, O.A., Scuseria, G.E., Constantin, L.A., Zhou, X., Burke, K., 2008. Restoring the densitygradient expansion for exchange in solids and surfaces. Phys. Rev. Lett., Vol. 100(13), 136406.

Roman, R.E., Cranford, S.W., 2014. Mechanical properties of silicene. Comp. Mat. Sci., Vol. 82, 50-55.

Roundy, D., Krenn, C.R., Cohen, M.L., Morris Jr, J.W., 2001. The ideal strength of tungsten. Phil. Mag. A, Vol. 81(7), 1725-1747. 
Saxena, S., Chaudhary, R.P., and Shukla, S., 2016. Stanene: Atomically Thick Free-standing Layer of 2D Hexagonal Tin. Scientific Report, Vol. 6, 31073.

Shodja, H.M., Delfani, M.R., 2011. A novel nonlinear constitutive relation for graphene and its consequence for developing closed-form expressions for Young's modulus and critical buckling strain of single-walled carbon nanotubes. Acta mech., Vol. 222(1-2), 91-101.

Shodja, H.M., Zaheri, A., Tehranchi, A., 2013. Ab initio calculations of characteristic lengths of crystalline materials in first strain gradient elasticity, Mech. Mat.,Vol. 61: 73-78.

Shodja, H.M., Tabatabaei, M., Esfarjani, K., 2014. First principles molecular dynamics studies of elastic constants, ideal tensile strength, chemistry of crack initiation, and surface and cohesive energies in amorphous silicon, Phil. Mag.,Vol. 94(25): 2913-2936.

Song, Z., Liu, C.C., Yang, J., Han, J., Ye, M., Fu, B., Yang, Y., Niu, Q., Lu, J., Yao, Y., 2014. Quantum spin Hall insulators and quantum valley Hall insulators of BiX/SbX (X equals; H, F, Cl and $\mathrm{Br}$ ) monolayers with a record bulk band gap. NPG Asia Materials, Vol. 6(12), e147.

Tabatabaei, M., Shodja, H.M., Ojaghnezhad, F., 2016. The role of strain on the quantum spin Hall effect and band inversion in stanene. Comp. Cond. Matter. http://dx.doi.org/10.1016/j.cocom.2016.10.003, In Press.

Topsakal, M., Cahangirov, S., Ciraci, S., 2010. The response of mechanical 
and electronic properties of graphane to the elastic strain. App. Phys. Lett. Vol. 96(9), 091912.

Van den Broek, B., Houssa, M., Scalise, E., Pourtois, G., Afanas'ev, V.V., Stesmans, A., 2014. Two-dimensional hexagonal tin: ab-initio geometry, stability, electronic structure and functionalization. 2D Materials, 1(2), 021004.

Webb, R., 2014. Future stuff: Stanene. New Scientist, Vol. 224(2990), 38-39.

Wei, X., Fragneaud, B., Marianetti, C.A., Kysar, J. W., 2009. Nonlinear elastic behavior of graphene: ab-initio calculations to continuum description. Phys. Rev. B, Vol. 80(20), 205407.

Xu, Y., Yan, B., Zhang, H.J., Wang, J., Xu, G., Tang, P., Duan, W., Zhang, S.C., 2013. Large-gap quantum spin Hall insulators in tin films. Phys. Rev. Lett., Vol. 111(13), 136804.

Yao, Y., Ye, F., Qi, X.L., Zhang, S.C., Fang, Z., 2007. Spin-orbit gap of graphene: First-principles calculations. Phys. Rev. B. Vol. 75, 041401(R).

Zhu, F., Chen, W.J., Xu, Y., Gao, C.L., Guan, D.D., Liu, C., Qian, D., Zhang, S., Jia, J.F., 2015. Epitaxial growth of two-dimensional stanene. Nature Materials, doi:10.1038/nmat4384.

Zhuang, H.L., Hennig, R.G., 2014. Computational discovery, characterization, and design of single-layer materials. JOM, Vol. 66(3), 366-374.

Zhang, R.W., Zhang, C.W., Ji, W.X., Li, S.S., Hu, S.J., Yan, S.S., Li, P., Wang P.J., and Li, F., 2015. Ethynyl-functionalized stanene film: a promis- 
ing candidate as large-gap quantum spin Hall insulator. New J. Phys. 17, 083036.

Zhang, R.W., Zhang, C.W., Ji, W.X., Li, S.S., Yan, S.S., Hu, S.J., Li, P., Wang, P.J., and Li, F., 2016. Room Temperature Quantum Spin Hall Insulator in Ethynyl-Derivative Functionalized Stanene Films. Scientific Reports. 6, 18879.

\section{Appendix A. The non-zero components of the elastic moduli ten- sors of $2 \mathrm{D}$ materials with hexagonal symmetry}

The second, third, fourth, and fifth order elastic moduli tensors associated with a general anisotropic three-dimensional hyper-elastic solid have, respectively, 21, 56, 126, and 252 independent elements. However, the nonzero components of these tensors for $2 \mathrm{D}$ materials correspond to $\mathrm{I}, \mathrm{J}=1,2$, and 6. It is well known that stanene is a $2 \mathrm{D}$ hexagonal lattice of the crystal class, $D_{6 h}$ possessing a six-fold rotation axis and six mirror planes perpendicular to the lattice plane. By applying all the symmetry elements of the crystal class, $D_{6 h}$, it is revealed that the elastic moduli tensors of second, third, fourth, and fifth order include, respectively, two, three, four, and five non-zero independent elastic constants. Thus, there are a total of fourteen independent elastic constants for stanene as summarized below

$$
\begin{aligned}
& C_{11}=C_{22}, C_{12}, \\
& C_{66}=\left(C_{11}-C_{12}\right) / 2, \\
& \text { in second order, } \\
& C_{111}, C_{112}, C_{222},
\end{aligned}
$$




$$
\begin{aligned}
& C_{122}=C_{111}-C_{222}+C_{112}, \\
& C_{166}=\left(3 C_{222}-2 C_{111}-C_{112}\right) / 4, \\
& C_{266}=\left(2 C_{111}-C_{222}-C_{112}\right) / 4,
\end{aligned}
$$

in third order,

$C_{1111}, C_{1112}, C_{1122}, C_{2222}$,

$C_{1166}=\left(-5 C_{1111}-4 C_{1112}+9 C_{2222}\right) / 24$,

$C_{1222}=\left(C_{1111}+2 C_{1112}-C_{2222}\right) / 2$,

$C_{1266}=\left(C_{1111}+2 C_{1112}-3 C_{1122}\right) / 12$,

$C_{2266}=\left(7 C_{1111}-4 C_{1112}-3 C_{2222}\right) / 24$,

$C_{6666}=\left(-C_{1111}-8 C_{1112}+6 C_{1122}+3 C_{2222}\right) / 16$,

in fourth order, and

$C_{11111}, C_{11112}, C_{11122}, C_{12222}, C_{22222}$,

$C_{11166}=\left(-4 C_{11111}-5 C_{11112}+9 C_{22222}\right) / 40$,

$C_{11222}=\left(C_{11111}+3 C_{11112}+2 C_{11122}-3 C_{12222}-C_{22222}\right) / 2$,

$C_{11266}=-\left(13 C_{11111}+30 C_{11112}+20 C_{11122}-45 C_{12222}-18 C_{22222}\right) / 120$,

$C_{12266}=\left(8 C_{11111}+15 C_{11112}-20 C_{11122}-3 C_{22222}\right) / 120$,

$C_{16666}=\left(11 C_{11111}+30 C_{11112}+10 C_{11122}-45 C_{12222}-6 C_{22222}\right) / 80$,

$C_{22266}=\left(9 C_{11111}-5 C_{12222}-4 C_{22222}\right) / 40$,

$C_{26666}=\left(-C_{11111}-30 C_{11112}+10 C_{11122}+15 C_{12222}+6 C_{22222}\right) / 80, \quad(\mathrm{~A} .1 \mathrm{~d})$ in fifth order. 